\title{
STUDY OF THE BOUNDARY LAYER AT SUPERSONIC SPEEDS IN TURBULENT FLOW: CASE OF FLOW ALONG A FLAT PLATE*
}

\author{
BY \\ CARLO FERRARI \\ Cornell Aeronautical Laboratory
}

\section{INTRODUCTION}

The flow of a gaseous fluid past a rigid, smooth half-plane, parallel to the asymptotic velocity of the stream, is studied. The edge of the half-plane (leading edge) is assumed to be orthogonal to the stream, and, in addition, it is assumed that the flow is everywhere "turbulent". The motion within the boundary layer is investigated in an attempt to clarify many of the assumed and conjectured facts relating to the turbulent layer within a supersonic stream. For instance, it is found for supersonic flow, within the range of Mach numbers examined, that a drag law holds which is formally identical with the one which is true for incompressible fluids, and, moreover, that the thickness of the boundary layer also obeys a law which is formally analogous to the one describing the growth of the layer in the incompressible fluid case. In addition, such relationships as those concerned with the velocity and temperature distributions within the layer, and the transfer of heat from the stream to the constraining plate at various Reynolds and Mach numbers are deduced from clearly set forth premises. Analogies and extensions are presented for full understanding of the phenomena described.

In presenting the results of this study of the motion within the turbulent layer it is found necessary to obtain the solution as an asymptotic one, i.e., one which is rigorously valid at a sufficient distance from the leading edge. The formulas obtained contain a parameter, the determination of which must be left to experiment. A plausible value of it, based upon the results obtained by Frössel ${ }^{1}$ from his investigation on sub- and supersonic streams in pipes, will be given.

\section{GENERAL EQUATIONS}

1. Symbols. Bars and primes will be used to denote, respectively, the mean values of the following quantities, and their fluctuations about their mean values due to turbulence $: i=$ enthalpy; $i_{0}=$ enthalpy of the external stream at zero speed; $i_{p}=$ enthalpy in contact with the wall; $i_{a}=$ enthalpy of the external undisturbed stream.

In the next set of symbols, the subscripts $0, p, a$, and the bars and primes give the quantities the same meanings as those specified above for the enthalpy: $T=$ absolute temperature; $U=$ component of the velocity along the $x$-axis; $V=$ component of the

*Received Dec. 20, 1948. Italian manuscript submitted to Cornell Aeronautical Laboratory July 22, 1948. This work was carried out under Navy BuOrd sponsorship.

${ }^{1}$ W. Frössel, Strömung in glatten geraden Rohren mit Ueber- und Unterschallgeschwindigkeit, Forsch. auf dem Geb. des Ing.-Wesens 7, 75-85 (1936). 
velocity along the $y$-axis; $W=\left(U^{2}+V^{2}\right)^{1 / 2} ; \rho=$ density of the fluid; $\mu=$ viscosity coefficient; $\nu=$ kinematic viscosity.

In addition, the following symbols are used: $p=$ pressure; $\tau=$ tangential friction stress; $\tau_{p}=$ tangential friction stress at the wall; $U^{*}=$ friction velocity $\left[U^{*}=\left(\tau_{p} / \rho_{p}\right)^{1 / 2}\right]$; $V_{l}=$ limit velocity of the undisturbed stream; $u_{a}=\bar{U}_{a} / V_{l} ; f=\bar{U} / U^{*} ; f_{a}=\bar{U}_{a} / U^{*}$; $\sigma=\bar{U} / \bar{U}_{a}=f / f_{a} ; u^{*}=U^{*} / V_{e} ; M_{a}=$ Mach number of the undisturbed stream; $\gamma=T_{0} / T_{p} ; \delta=$ thickness of the boundary layer; $t=$ time; $c_{r}=$ coefficient of local friction drag $\left(c_{r}=2 \tau_{p} / \rho_{a} \bar{U}_{a}^{2}\right) ; C_{r}=$ coefficient of total friction drag for a plate of length $x ; q=$ amount of heat transferred through the plate from the fluid, per unit length of wall.

The meaning of other symbols used in this paper will be given as they appear in the text.

2. Equations of motion and of continuity. In the region outside the laminar sublayer one may neglect the transport of momentum due to thermic molecular vibration in comparison with that due to turbulence. Let the $x$-axis coincide with the line of intersection of the rigid plate with the plane on which the motion is being studied. Then one may write:

$$
\begin{aligned}
& -\frac{\partial p}{\partial x}=\frac{\partial}{\partial t}(\rho U)+\frac{\partial}{\partial x}\left(\rho U^{2}\right)+\frac{\partial}{\partial y}(\rho U V), \\
& -\frac{\partial p}{\partial y}=\frac{\partial}{\partial t}(\rho V)+\frac{\partial}{\partial x}(\rho U V)+\frac{\partial}{\partial y}\left(\rho V^{2}\right) .
\end{aligned}
$$

Let $p=\bar{p}+p^{\prime}, \rho=\bar{\rho}+\rho^{\prime}, U=\bar{U}+U^{\prime}$, and $V=\bar{V}+V^{\prime}$. Then at a sufficiently large distance from the leading edge of the plate it is true, in all likelihood, that $\bar{\rho}=\bar{\rho}(y)$, $\bar{U}=\bar{U}(y)$ and $\bar{V}=0$. Therefore, if one neglects, as is usually done, $\overline{\rho V^{2}}$ in comparison with $\overline{\rho U V}$, it is found that

$$
\begin{aligned}
& \overline{\rho U V}=\overline{\rho U^{\prime} V^{\prime}}+\overline{U \rho^{\prime} V^{\prime}}+\overline{\rho^{\prime} U^{\prime} V^{\prime}}=\tau=\text { const. }=\tau_{p}, \\
& \bar{p}=\text { const. }=\bar{p}_{a} .
\end{aligned}
$$

It certainly seems allowable to admit that $\overline{\rho^{\prime} U^{\prime} V^{\prime}}$ may be neglected in comparison with the other terms; then one can substitute the simplification

$$
\overline{\rho U^{\prime} V^{\prime}}+\overline{U_{\rho}^{\prime} V^{\prime}}=\tau_{p}
$$

for the first of Eqs. (2).

From the equation of continuity,

$$
\frac{\partial \rho}{\partial t}+\frac{\partial}{\partial x}(\rho U)+\frac{\partial}{\partial y}(\rho V)=0,
$$

the following relation may be established:

$$
\overline{\rho^{\prime} V^{\prime}}=c \overline{\rho_{p}} U^{*},
$$

where $c$ is a parameter, related to the Mach number and concerning which we know solely that

$$
\lim c=0 \quad \text { as } \quad M_{a} \rightarrow 0 .
$$


3. Energy equation. If in addition to neglecting $\overline{\rho^{\prime} U^{\prime} V^{\prime}}$ one neglects, as in Sec. 2 , the viscosity and the molecular conductivity in the region outside of the laminar sublayer, one easily may find that

$$
c_{\mathrm{v}} \frac{d T}{d t}+\frac{d}{d t}\left(\frac{p}{\rho}\right)=\frac{1}{\rho}\left(\frac{\partial p}{\partial t}+U \frac{\partial p}{\partial x}+V \frac{\partial p}{\partial y}\right),
$$

where $c_{v}$ denotes the specific heat at constant volume. From this equation we obtain

$$
\rho \frac{d}{d t}\left(i+\frac{1}{2} W^{2}\right)=\frac{\partial p}{\partial t} .
$$

But

$$
\begin{aligned}
\rho \frac{d}{d t}\left(i+\frac{1}{2} W^{2}\right)=\frac{\partial}{\partial x}\left[\rho U\left(i+\frac{1}{2} W^{2}\right)\right] & +\frac{\partial}{\partial y}\left[\rho V\left(i+\frac{1}{2} W^{2}\right)\right] \\
& +\frac{\partial}{\partial t}\left[\rho\left(i+\frac{1}{2} W^{2}\right)\right],
\end{aligned}
$$

and, therefore, at a sufficiently large distance from the leading edge, one has

$$
\overline{\rho V\left(i+\frac{1}{2} W^{2}\right)}=\sim \bar{\rho} \overline{V^{\prime}\left(i+\frac{1}{2} W^{2}\right)^{\prime}}+\left(\bar{i}+\frac{1}{2} \bar{U}^{2}\right) \overline{\rho^{\prime}} \bar{V}^{\prime}=\text { const. }=a \bar{\rho}_{p} U^{* 3} .
$$

4. Explicit form of the terms defining the turbulent transport of momentum and energy. In order to describe the terms which define the turbulent transport of momentum and energy as functions of the geometric and kinetic characteristics of the average motion, we may go back to the well-known considerations which permit the establishment of the following expressions for these terms: ${ }^{2}$

$$
\overline{U^{\prime} V^{\prime}}=l \frac{d \bar{U}}{d y}\left(\overline{V^{\prime 2}}\right)^{1 / 2}, \quad \overline{V^{\prime}\left(i+\frac{1}{2} W^{2}\right)^{\prime}}=l_{1} \frac{d}{d y}\left(\bar{i}+\frac{1}{2} \bar{U}^{2}\right)\left(\overline{\left.V^{\prime 2}\right)^{1 / 2}},\right.
$$

where $l$ and $l_{1}$ are lengths which are likely to be related to the scale of turbulence. It appears plausible to put $l=l_{1}$. In fact if

$$
\bar{i}+\frac{1}{2} \bar{U}^{2}=\text { const. }
$$

(the flow is everywhere isoenergetic), the correlation between $V^{\prime}$ and $\left(i+W^{2} / 2\right)^{\prime}$ may be assumed equal to zero, and therefore $\overline{V^{\prime}\left(i+W^{2} / 2\right)^{\prime}}=0$. On the other hand the following relations hold:

$$
\begin{aligned}
\overline{V^{\prime}\left(i+\frac{1}{2} W^{2}\right)^{\prime}} & =\overline{V^{\prime} i^{\prime}}+\overline{\frac{1}{2} V^{\prime} W^{\prime 2}}=\overline{V^{\prime} i^{\prime}}+\overline{\frac{1}{2} V^{\prime}\left(2 \bar{U} U^{\prime}+{U^{\prime}}^{2}+V^{\prime 2}\right)} \\
& =\sim \overline{V^{\prime} i^{\prime}}+\bar{U} \overline{V^{\prime} U^{\prime}}=l_{2} \frac{d \bar{i}}{d y}\left(\overline{V^{\prime 2}}\right)^{1 / 2}+\bar{U} l \overline{\left(V^{\prime 2}\right)^{1 / 2}} \frac{d \bar{U}}{d y} \\
& =\left(\overline{V^{\prime 2}}\right)^{1 / 2}\left(l_{2} \frac{d \bar{i}}{d y}+\frac{1}{2} l \frac{d \bar{U}^{2}}{d y}\right)=0,
\end{aligned}
$$

${ }^{2} \mathrm{G}$. I. Taylor, The transport of vorticity and heat through fluids in turbulent motion, Proc. Roy. Soc. London (A) 135, 685-705 (1932). 
where the meaning of the symbols is obvious. Now, in order that Eq. $\left(6^{\prime}\right)$ be a necessary consequence of Eq. (6), whatever the particular variation of $\bar{i}$ and $\bar{U}$ in the region may be, it suffices that $l_{2}=l$ everywhere, and therefore also that $l_{1}=l$.

\section{VELOCITY AND TEMPERATURE WITHIN THE BOUNDARY LAYER}

5. Law of variation of temperature within the boundary layer. Taking into account Eq. $\left(3^{\prime}\right)$ and Eq. (5), Eqs. $\left(2^{\prime}\right)$ and $\left(4^{\prime}\right)$ become

$$
\begin{aligned}
& \bar{\rho} l \frac{d \bar{U}}{d y}\left(\overline{V^{\prime 2}}\right)^{1 / 2}+c \overline{\rho_{p}} U^{*} \bar{U}=\bar{\rho}_{p} U^{* 2} \\
& \bar{\rho} l \frac{d}{d y}\left(\bar{i}+\frac{1}{2} \bar{U}^{2}\right)\left(\overline{V^{\prime 2}}\right)^{1 / 2}+\left(\bar{i}+\frac{1}{2} \bar{U}^{2}\right) c \bar{\rho}_{p} U^{*}=a \bar{\rho}_{p} U^{* 3} .
\end{aligned}
$$

One obtains from them that

$$
\bar{i}+\frac{1}{2} \bar{U}^{2}=\overline{i_{p}}+B \bar{U},
$$

where $\bar{i}_{p}$ is the enthalpy at the wall, while $B$ is related to the parameters $a$ and $c$ through the equation

$$
B+c \frac{\overline{i_{p}}}{U^{*}}-a U^{*}=0 .
$$

Now, at the edge of the boundary layer, where $\bar{i}=\bar{i}_{a}, \bar{U}=\bar{U}_{a}$, one must have $\bar{i}_{a}+$ $\bar{U}_{a}^{2} / 2=\bar{i}_{p}+B \bar{U}_{a}$. It follows immediately that

$$
\begin{aligned}
B & =\frac{\bar{i}_{a}+\bar{U}_{a}^{2} / 2-\bar{i}_{p}}{\bar{U}_{a}}=\frac{i_{0}-\bar{i}_{p}}{\bar{U}_{a}}=\frac{i_{0}}{\bar{U}_{a}}\left(1-\frac{1}{\gamma}\right) \\
& =\frac{\gamma-1}{\gamma} \bar{U}_{a}\left[\frac{1}{2}+\frac{1}{(k-1) M_{a}^{2}}\right],
\end{aligned}
$$

where $k$ is the adiabatic exponent and $\gamma=T_{0} / T_{p}$. The law of variation of temperature in the inside of the boundary layer is therefore obtained as

$$
\begin{aligned}
\frac{\bar{T}}{T_{0}} & =\frac{1}{\gamma}-\frac{\bar{U}^{2}}{\bar{U}_{a}^{2}} \frac{(k-1) M_{a}^{2}}{2+(k-1) M_{a}^{2}}+\frac{\gamma-1}{\gamma}\left[\frac{1}{2}+\frac{1}{(k-1) M_{a}^{2}}\right] \frac{\bar{U}}{\bar{U}_{a}} \frac{2(k-1) M_{a}^{2}}{2+(k-1) M_{a}^{2}} \\
& =\frac{1}{\gamma}+\frac{\bar{U}}{\bar{U}_{a}}\left[\frac{\gamma-1}{\gamma}-\frac{(k-1) M_{a}^{2}}{2+(k-1) M_{a}^{2}} \frac{\bar{U}}{\bar{U}_{a}}\right] .
\end{aligned}
$$

This relation is identical with that defining the law of variation of $\bar{T}$ within the boundary layer in the case of laminar flow when the Prandtl number is equal to unity ${ }^{3}, \operatorname{Pr}=1$.

6. Law of variation of velocity within the boundary layer.

6.1. Integration of the equation of motion. In order to carry on this discussion further the quantity $l\left(\overline{V^{\prime 2}}\right)^{1 / 2}$, denoting the so-called coefficient of turbulent transport, must

${ }^{3}$ Th. v. Kármán and H. S. Tsien, Boundary layer in compressible fluids, J. Aero. Sci. 5, 228 (1938). 
be given an explicit form. Now the same formulation will be assumed for this coefficient that is commonly accepted in the case of incompressible fluid flow (one might repeat here the reasons which justify it). ${ }^{4}$

Let, therefore,

$$
l\left(\bar{V}^{\prime 2}\right)^{1 / 2}=U^{*} y \Phi\left(f, f^{\prime}, \cdots\right)=U^{*} y\left(\alpha_{0}+\alpha_{1} f+\alpha_{2} f^{\prime}+\cdots\right),
$$

where $f=\bar{U} / U^{*}$ while $f^{\prime}=d f / d \eta$ and $\alpha_{0}, \alpha_{1}, \alpha_{2}, \cdots$ are a priori unknown functions of $\eta=y / \delta$, if $\delta$ is the thickness of the boundary layer.

Accepting the same degree of approximation as in the case of incompressible fluids, let

$$
l\left(\overline{V^{\prime 2}}\right)^{1 / 2}=U^{*} y \alpha_{0},
$$

where $\alpha_{0}$ is a constant. Hence

$$
l\left(\bar{V}^{\prime 2}\right)^{1 / 2} \frac{d \bar{U}}{d y}=U^{* 2} \eta f^{\prime} \alpha_{0} .
$$

Now, since it is true that

$$
\begin{aligned}
\frac{\bar{\rho}}{\bar{\rho}_{p}} & =\frac{\bar{T}_{p}}{\bar{T}}=\frac{\bar{i}_{p}}{i_{p}-\bar{U}^{2} / 2+B \bar{U}}=\left[1-\frac{1}{2} \frac{\bar{U}^{2}}{\bar{U}_{a}^{2}} \frac{\bar{U}_{a}^{2}}{i_{0}} \frac{i_{0}}{\bar{i}_{p}}+\frac{\bar{U}}{\bar{U}_{a}}\left(\frac{i_{0}}{\bar{i}_{p}}-1\right)\right]^{-1} \\
& =\frac{1}{1-u_{a}^{2} \gamma \sigma^{2}+(\gamma-1) \sigma},
\end{aligned}
$$

the first of Eqs. (7) becomes

$$
\left\{\bar{\rho}_{p} U^{*^{2}} /\left[1-u_{a}^{2} \gamma \sigma^{2}+(\gamma-1) \sigma\right]\right\} \alpha_{0} \eta f^{\prime}+c \bar{\rho}_{p} U^{*^{2}} f=\bar{\rho}_{p} U^{*^{2}}
$$

or also

$$
\alpha_{0} \eta f^{\prime}+c f\left[1-\gamma u^{*^{2}} f^{2}+\frac{f}{f_{a}}(\gamma-1)\right]=1-\gamma u^{*^{2}} f^{2}+\frac{f}{f_{a}}(\gamma-1) .
$$

By separation of variables one obtains

$$
\frac{\alpha_{0} d f}{\left[1-\gamma u^{*^{2}} f^{2}+(\gamma-1) f / f_{a}\right](1-c f)}=\frac{d \eta}{\eta} .
$$

Let

$$
\begin{aligned}
& f_{1}=\frac{\gamma-1}{2 \gamma^{1 / 2}} \frac{1}{u_{a}}\left[1 \pm\left(1+\frac{4 \gamma}{(\gamma-1)^{2}} u_{a}^{2}\right)^{1 / 2}\right], \\
& f_{2}=-\frac{\gamma-1}{2 \gamma^{1 / 2}} \frac{1}{u_{a}}\left[1 \mp\left(1+\frac{4 \gamma}{(\gamma-1)^{2}} u_{a}^{2}\right)^{1 / 2}\right],
\end{aligned}
$$

where one employs either the upper or the lower sign before the radical according to whether $\gamma \geq 1$ or $\gamma<1$. Then we have

${ }^{4}$ G. Moretti, La teoria del trasporto turbolento, L'Aerotecnica 27, 44-50 (1947), and Considerazioni sullo studio della turbolenza nei tubi e nei canali, L'Aerotecnica 27, 404-410 (1947); or C. Ferrari, Lo stato attuale della teoria della turbolenza, Lectures on Phys. and Math. in Univ. of Torino, 1936, p. 223. 


$$
\begin{aligned}
\frac{1}{\left[1-\gamma u^{* 2} f^{2}+(\gamma-1) f / f_{a}\right](1-c f)} & =\frac{1}{\left(f_{1}-\gamma^{1 / 2} u^{*} f\right)\left(\gamma^{1 / 2} u^{*} f+f_{2}\right)(1-c f)} \\
& =\frac{a_{1}}{f_{1}-\gamma^{1 / 2} u^{*} f}+\frac{a_{2}}{\gamma^{1 / 2} u^{*} f+f_{2}}+\frac{a_{3}}{1-c f},
\end{aligned}
$$

where

$$
\begin{gathered}
a_{1}=\frac{\gamma^{1 / 2} u^{*}}{\left(f_{1}+f_{2}\right)\left(\gamma^{1 / 2} u^{*}-c f_{1}\right)}, \quad a_{2}=\frac{\gamma^{1 / 2} u^{*}}{\left(f_{1}+f_{2}\right)\left(\gamma^{1 / 2} u^{*}+c f_{2}\right)}, \\
a_{3}=\frac{-c^{2}}{\left(\gamma^{1 / 2} u^{*}+c f_{2}\right)\left(\gamma^{1 / 2} u^{*}-c f_{1}\right)} .
\end{gathered}
$$

One should observe that within the boundary layer $f / f_{a} \leq 1$, while $f_{1} / f_{a} \gamma^{1 / 2} u^{*}>1$, $-f_{2} / f_{a} \gamma^{1 / 2} u^{*}<0$, for any value of $\gamma$ whatever; thus the following inequalities certainly hold: $-f_{2} / f_{a} \gamma^{1 / 2} u^{*}<f / f_{a}<f_{1} / f_{a} \gamma^{1 / 2} u^{*}$. One has, besides, that $f / f_{a} \neq 1 / c f_{a}$; and this relation, which may now be accepted as a restriction which the constant $c$ must satisfy, will be justified later in Sec. 10.2 .

Thus it appears that the function of $f$ appearing in the first member of Eq. (16) has no singularities within the layer. By integrating Eq. (16) one obtains the equation

$$
\begin{aligned}
& \alpha_{0}\left[-\frac{a_{1}}{\gamma^{1 / 2} u^{*}} \log \left(f_{1}-\gamma^{1 / 2} u^{*} f\right)+\frac{a_{2}}{\gamma^{1 / 2} u^{*}} \log \left(\gamma^{1 / 2} u^{*} f+f_{2}\right)-\frac{a_{3}}{c} \log (1-c f)\right] \\
& \quad=\log \eta+\log A,
\end{aligned}
$$

where $\log A$ denotes a suitable constant of integration.

6.2. Determination of the constant of integration. Equation (19) describes the law of variation of the mean velocity in the inside of the turbulent boundary layer, and, therefore, for all values of $f$ included within the interval, $f_{e} \leq f \leq f_{a}$, where $f_{e}$ denotes the value of $f$ at the boundary of the so-called laminar sublayer. ${ }^{5}$ For values of $f$ close to $f_{\text {。 }}$, for which one has $\gamma^{1 / 2} u^{*} f \ll f_{1}$ and $\gamma^{1 / 2} u^{*} f \ll f_{2}$, it is permissible to put

$$
\begin{gathered}
\log \left(f_{1}-\gamma^{1 / 2} u^{*} f\right) \cong \log f_{1}-\gamma^{1 / 2} u^{*} \frac{f}{f_{1}}, \quad \log \left(\gamma^{1 / 2} u^{*} f+f_{2}\right) \cong \log f_{2}+\gamma^{1 / 2} u^{*} \frac{f}{f_{2}}, \\
\log (1-c f) \cong-c f .
\end{gathered}
$$

Taking into account Eqs. (18), one may put Eq. (19) in the form

$$
\begin{gathered}
\frac{\alpha_{0}}{c}\left[-\left(\frac{\gamma^{1 / 2}}{c} u^{*}+f_{2}\right)\left(\log f_{1}-\gamma^{1 / 2} u^{*} \frac{f}{f_{1}}\right)+\left(\frac{\gamma^{1 / 2}}{c} u^{*}-f_{1}\right)\left(\log f_{2}+\gamma^{1 / 2} u^{*} \frac{f}{f_{2}}\right)\right. \\
\left.-\left(f_{1}+f_{2}\right) c f\right]=\left(f_{1}+f_{2}\right)\left(\frac{\gamma^{1 / 2}}{c} u^{*}+f_{2}\right)\left(\frac{\gamma^{1 / 2}}{c} u^{*}-f_{1}\right)(\log \eta+\log A) .
\end{gathered}
$$

At the boundary of the sublayer, the thickness of which will be denoted by $y_{0}$, one has, therefore, 


$$
\begin{aligned}
& \frac{\alpha_{0}}{c}\left[\frac{\gamma}{c} u^{*^{2}} \frac{f_{1}+f_{2}}{f_{1} f_{2}}+\gamma^{1 / 2} u^{*} \frac{f_{2}^{2}-f_{1}^{2}}{f_{1} f_{2}}-c\left(f_{1}+f_{2}\right)\right] f_{\bullet} \\
& +\frac{\alpha_{0}}{c}\left[-\left(\gamma^{1 / 2} \frac{u^{*}}{c}+f_{2}\right) \log f_{1}+\left(\gamma^{1 / 2} \frac{u^{*}}{c}-f_{1}\right) \log f_{2}\right] \\
& \quad=\left(f_{1}+f_{2}\right)\left(\gamma^{1 / 2} \frac{u^{*}}{c}+f_{2}\right)\left(\gamma^{1 / 2} \frac{u^{*}}{c}-f_{1}\right)\left(\log \frac{y_{e}}{\delta}+\log A\right) .
\end{aligned}
$$

And as $f_{1} f_{2}=1$, there is obtained also:

$$
\begin{aligned}
\alpha_{0}\left(f_{1}+f_{2}\right) & {\left[\frac{\gamma}{c^{2}} u^{*^{2}}+\frac{\gamma^{1 / 2}}{c} u^{*}\left(f_{1}-f_{2}\right)-1\right] f_{c} } \\
+\frac{\alpha_{0}}{c}[ & \left.-\left(\gamma^{1 / 2} \frac{u^{*}}{c}+f_{2}\right) \log f_{1}+\left(\gamma^{1 / 2} \frac{u^{*}}{c}-f_{1}\right) \log f_{2}\right] \\
& =\left(f_{1}+f_{2}\right)\left(\gamma^{1 / 2} \frac{u^{*}}{c}+f_{2}\right)\left(\gamma^{1 / 2} \frac{u^{*}}{c}-f_{1}\right)\left(\log \frac{y_{e}}{\delta}+\log A\right) \\
& =\left(f_{1}+f_{2}\right)\left[\frac{\gamma}{c^{2}} u^{*^{2}}+\gamma^{1 / 2} \frac{u^{*}}{c}\left(f_{2}-f_{1}\right)-1\right]\left(\log \frac{y_{e}}{\delta}+\log A\right),
\end{aligned}
$$

whence

$$
\begin{aligned}
f_{\iota}=\frac{1}{\alpha_{0}} & \left(\log \frac{y_{e}}{\delta}+\log A\right)-\frac{1}{c} \frac{1}{f_{1}+f_{2}}\left[-\left(\gamma^{1 / 2} \frac{u^{*}}{c}+f_{2}\right) \log f_{1}+\left(\gamma^{1 / 2} \frac{u^{*}}{c}\right.\right. \\
& \left.\left.-f_{1}\right) \log f_{2}\right] \frac{1}{\left(\gamma^{1 / 2} u^{*} / c+f_{2}\right)\left(\gamma^{1 / 2} u^{*} / c-f_{1}\right)}=\frac{1}{\alpha_{0}}\left(\log \frac{y_{e}}{\delta}+\log A\right) \\
& -\frac{1}{c\left(f_{1}+f_{2}\right)}\left(-\frac{1}{\gamma^{1 / 2} u^{*} / c-f_{1}} \log f_{1}+\frac{1}{\gamma^{1 / 2} u^{*} / c+f_{2}} \log f_{2}\right)=\frac{y_{e} U^{*}}{\nu_{p}},
\end{aligned}
$$

where it is assumed, as is done by Kármán ${ }^{8}$, that within the sublayer the velocity is a linear function of $y$. Thus the following equation may be derived:

$\log A=-\log \frac{y_{e}}{\delta}+\alpha_{0} \frac{y_{e} U^{*}}{\nu_{p}}+\frac{\alpha_{0}}{c\left(f_{1}+f_{2}\right)}\left(-\frac{\log f_{1}}{\gamma^{1 / 2} u^{*} / c-f_{1}}+\frac{\log f_{2}}{\gamma^{1 / 2} u^{*} / c+f_{2}}\right)$.

When Eq. (21) is inserted into Eq. (19), we find the relation

$$
\begin{aligned}
\frac{\alpha_{0}}{c}[ & -\frac{1}{\left(f_{1}+f_{2}\right)\left(\gamma^{1 / 2} u^{*} / c-f_{1}\right)} \log \left(1-\gamma^{1 / 2} u^{*} \frac{f}{f_{1}}\right) \\
& +\frac{1}{\left(f_{1}+f_{2}\right)\left(\gamma^{1 / 2} u^{*} / c+f_{2}\right)} \log \left(1+\gamma^{1 / 2} u^{*} \frac{f}{f_{2}}\right) \\
& \left.+\frac{1}{\left(\gamma^{1 / 2} u^{*} / c+f_{2}\right)\left(\gamma^{1 / 2} u^{*} / c-f_{1}\right)} \log (1-c f)\right]=\log y^{*}+B
\end{aligned}
$$


where

$$
y^{*}=\frac{y U^{*}}{\nu_{p}} \quad \text { and } \quad B=\alpha_{0} \frac{y_{e} U^{*}}{\nu_{p}} \quad \text { with } \quad \nu_{p}=\frac{\mu_{p}}{\rho_{p}}
$$

It is assumed here, as in the study of incompressible fluid flow, that $\alpha_{0}$ and $y_{e} U^{*} / \nu_{p}$ are universal constants, which are independent, in particular, of the value of $\gamma$. It should be observed that for $\gamma=1$ one has $f_{1}=f_{2}=1$. Thus Eq. (19') leads, for the value of $\log A$ given by Eq. (21), to the following fact:

$$
f=\frac{1}{\alpha_{0}}\left(\log y^{*}+B\right)
$$

This relation is identical with that holding for incompressible fluid flows. This must be true not only because the equations established in the case of incompressibility must be deducible as a particular case from those which are true for gases, but also because for $\gamma=1$, in proximity to the walls, the effect of compressibility is negligible.

Thus it seems allowable to assume in every case, for $\alpha_{0}$ as well as for $B$, the values obtained from experiment for $\rho=$ const.

\section{DRAG LAW AND LAW OF VARIATION OF THE THICKNESS OF THE BOUNDARY LAYER}

7. Dependence of the local drag coefficient upon the local Reynolds number. Equation (22) gives, as already said, the law of variation of the velocity outside of and on the boundary of the laminar sublayer, i.e., for $y \geq y_{e}$. By following a procedure identical with that used by Prandtl ${ }^{6}$ it is, however, possible to extend the applicability of this law right up to the wall and thus evaluate the coefficient of local resistance, $c_{r}=2 \tau_{p} / \rho_{a} \bar{U}_{a}^{2}$. To this purpose, a transformation of coordinates is performed such that for $y^{*}=0, f=0$. One need only substitute $\log \left(1+b y^{*}\right)$ for $\log y^{*}+B$. When this is done, it may easily be verified that for suitable values of $b$ and for $y^{*}$ sufficiently large the new expression is practically coincident with the old one, and that the new expression vanishes for $y^{*}=0$. Equation (22) thus becomes

$$
\begin{aligned}
\frac{\alpha_{0}}{c}[ & -\frac{1}{\left(f_{1}+f_{2}\right)\left(\gamma^{1 / 2} u^{*} / c-f_{1}\right)} \log \left(1-\gamma^{1 / 2} u^{*} \frac{f}{f_{1}}\right) \\
& +\frac{1}{\left(f_{1}+f_{2}\right)\left(\gamma^{1 / 2} u^{*} / c+f_{2}\right)} \log \left(1+\gamma^{1 / 2} u^{*} \frac{f}{f_{2}}\right) \\
& \left.+\frac{1}{\left(\gamma^{1 / 2} u^{*} / c+f_{2}\right)\left(\gamma^{1 / 2} u^{*} / c-f_{1}\right)} \log (1-c f)\right]=\log \left(1+b y^{*}\right) .
\end{aligned}
$$

'L. Prandtl, "The mechanics of viscous fluids," Aerodynamic Theory, ed. by W. F. Durand, vol. 3 Julius Springer, Berlin, 1934, p. 145. 
Equation (22') may be used in order to express the coefficient $c_{r}$ in terms of the Reynolds number, $R_{x}=\bar{U}_{a} x / \nu_{p}$, by means of

$$
\tau_{p}=\frac{d}{d x} \int_{0}^{\delta} \bar{\rho} \bar{U}\left(\bar{U}_{a}-\bar{U}\right) d y .
$$

The method explained hereafter is similar to that used by Prandtl. ${ }^{6}$ Remembering that $\tau_{p}=\bar{\rho}_{p}\left(\bar{U}_{a}^{2} / f_{a}^{2}\right), d y=\left(\nu_{p} / U^{*}\right) d y^{*}$ and putting $\delta^{*}=U^{*} \delta / \nu_{p}$, we find from Eq. (23) that

$$
\bar{\rho}_{p} \frac{\bar{U}_{a}^{2}}{f_{a}^{2}}=\bar{\rho}_{p} \nu_{p} \bar{U}_{a} \frac{d}{d x} \int_{0}^{\delta^{*}} \frac{\bar{\rho}}{\bar{\rho}_{p}} f(1-\sigma) d y^{*}=\bar{\rho}_{p} \nu_{p} \bar{U}_{a} \frac{d}{d x} \int_{0}^{1} \frac{\bar{\rho}}{\bar{\rho}_{p}} f(1-\sigma) \frac{d y^{*}}{d \sigma} d \sigma .
$$

Now from Eq. $\left(22^{\prime}\right)$ one obtains

$$
\begin{aligned}
1+b y^{*}=( & \left.\frac{1}{1-}-\frac{\gamma^{1 / 2} u^{*} f / f_{2}}{\gamma^{1 / 2} u^{*} f / f_{1}}\right)^{\alpha_{0} / c f^{*}\left(\gamma^{1 / 2} u^{*} / c+f_{2}\right)} \\
& \times\left(\frac{1-c f}{1-\gamma^{1 / 2} u^{*} f / f_{1}}\right)^{\alpha_{0} / c\left(\gamma^{1 / 2} u^{*} / c+f_{2}\right)\left(\gamma^{1 / 2} u^{*} / c-f_{1}\right)},
\end{aligned}
$$

where

$$
f^{*}=f_{1}+f_{2}
$$

Let

$$
c=\beta \frac{U^{*}}{V_{l}}=\beta \frac{u_{a}}{f_{a}},
$$

where $\beta$ is a numerical parameter, a priori unknown. It should be noted that the relationship (26) represents merely a heuristic hypothesis, which is the most simple representation that may be formulated in connection with condition $\left(3^{\prime}\right)$; but nothing justifies $a$ priori the assumption made in this paper of regarding $\beta$ as constant. Equation (24) can be written in the form

$$
\begin{aligned}
1+b y^{*}=( & \left.\frac{1+\gamma^{1 / 2} \sigma u_{a} / f_{2}}{1-\gamma^{1 / 2} \sigma u_{a} / f_{1}}\right)^{\alpha_{0} f_{a} / u_{a} \sigma^{*}\left(\gamma^{1 / 2}+\beta f_{a}\right)} \\
& \quad \times\left(\frac{1-\beta u_{a} \sigma}{1-\gamma^{1 / 2} \sigma u_{a} / f_{1}}\right)^{\alpha_{0} \beta f_{a} / u_{a}\left(\gamma^{1 / 2}+\beta f_{a}\right)\left(\gamma^{1 / 2}-\beta f_{1}\right)} .
\end{aligned}
$$

By differentiating Eq. $\left(22^{\prime}\right)$ one obtains

$$
\begin{aligned}
\frac{b d y^{*}}{1+b y^{*}=} \frac{\alpha_{0}}{c}\left[\frac{1}{f^{*}\left(\gamma^{1 / 2} u^{*} / c-f_{1}\right)} \frac{\gamma^{1 / 2} u^{*}}{f_{1}-\gamma^{1 / 2} u^{*} f}+\frac{1}{f^{*}\left(\gamma^{1 / 2} u^{*} / c+f_{2}\right)} \frac{\gamma^{1 / 2} u^{*}}{\gamma^{1 / 2} u^{*} f+f_{2}}\right. \\
\\
\left.\quad-\frac{c}{\left(\gamma^{1 / 2} u^{*} / c+f_{2}\right)\left(\gamma^{1 / 2} u^{*} / c-f_{1}\right)} \frac{1}{1-c f}\right] d f \\
=\frac{\alpha_{0} d f}{\left(f_{1}-\gamma^{1 / 2} u^{*} f\right)\left(\gamma^{1 / 2} u^{*} f+f_{2}\right)(1-c f)} \\
=\frac{\alpha_{0} f_{a} d \sigma}{\left[1-\gamma u_{a}^{2} \sigma^{2}+(\gamma-1) \sigma\right]\left(1-\beta u_{a} \sigma\right)}
\end{aligned}
$$


so that taking Eq. $\left(24^{\prime}\right)$ into account, it is found that

$$
\begin{aligned}
\frac{d y^{*}}{d \sigma}=\frac{\alpha_{0}}{b} & f_{a} \frac{1}{\left[1-\gamma u_{a}^{2} \sigma^{2}+(\gamma-1) \sigma\right]\left(1-\beta u_{a} \sigma\right)} \\
& \times\left(\frac{1+\gamma^{1 / 2} \sigma u_{a} / f_{2}}{1-\gamma^{1 / 2} \sigma u_{a} / f_{1}}\right)^{\alpha_{1} f_{a}}\left(\frac{1-\beta u_{a} \sigma}{1-\gamma^{1 / 2} \sigma u_{a} / f_{1}}\right)^{\alpha_{2} f_{a}},
\end{aligned}
$$

in which

$$
\alpha_{1}=\alpha_{0} / f^{*} u_{a}\left(\gamma^{1 / 2}+\beta f_{2}\right), \quad \alpha_{2}=\alpha_{0} \beta / u_{a}\left(\gamma^{1 / 2}+\beta f_{2}\right)\left(\gamma^{1 / 2}-\beta f_{1}\right) .
$$

By inserting into Eq. $\left(23^{\prime}\right)$ the expression for $\bar{\rho} / \bar{\rho}_{p}$ given by Eq. (14), one obtains

$$
\begin{aligned}
\frac{\bar{U}_{a}}{\nu_{p} f_{a}^{2}}=\frac{\alpha_{0}}{b} & \frac{d}{d x} \\
& {\left[f_{a}^{2} \int_{0}^{1} \frac{\sigma(1-\sigma)}{\left[1-u_{a}^{2} \gamma \sigma^{2}+(\gamma-1) \sigma\right]^{2}} \frac{1}{\left(1-\beta u_{a} \sigma\right)}\right.} \\
& \left.\times\left(\frac{1+\gamma^{1 / 2} \sigma u_{a} / f_{2}}{1-\gamma^{1 / 2} \sigma u_{a} / f_{1}}\right)^{\alpha_{1} f_{a}}\left(\frac{1-\beta u_{a} \sigma}{1-\gamma^{1 / 2} \sigma u_{a} / f_{1}}\right)^{\alpha_{2} f_{a}} d \sigma\right] .
\end{aligned}
$$

By differentiation it is found that

$$
\begin{aligned}
\frac{\bar{U}_{a}}{\nu_{p} f_{a}^{2}}=\frac{\alpha_{0}}{b} & \frac{d f_{a}}{d x} f_{a} \int_{0}^{1} \frac{\sigma(1-\sigma)}{\left[1-u_{a}^{2} \gamma \sigma^{2}+(\gamma-1) \sigma\right]^{2}\left(1-\beta u_{a} \sigma\right)}\left(\frac{1+\gamma^{1 / 2} \sigma u_{a} / f_{2}}{1-\gamma^{1 / 2} \sigma u_{a} / f_{1}}\right)^{\alpha_{1} f_{a}} \\
& \times\left(\frac{1-\beta u_{a} \sigma}{1-\gamma^{1 / 2} \sigma u_{a} / f_{1}}\right)^{\alpha_{2} f_{a}}\left[2+f_{a} \alpha_{1} \log \frac{1+\gamma^{1 / 2} \sigma u_{a} / f_{2}}{1-\gamma^{1 / 2} \sigma u_{a} / f_{1}}\right. \\
& \left.+f_{a} \alpha_{2} \log \frac{1-\beta u_{a} \sigma}{1-\gamma^{1 / 2} \sigma u_{a} / f_{1}}\right] d \sigma=\frac{\alpha_{0}}{b} f_{a} \frac{d f_{a}}{d x} \varphi_{0}\left(f_{a}\right)
\end{aligned}
$$

whence

$$
d R_{x}=\frac{\bar{U}_{a} d x}{\nu_{p}}=\frac{\alpha_{0}}{b} f_{a}^{3} \varphi_{0}\left(f_{a}\right) d f_{a}=\frac{\alpha_{0}}{b} \varphi_{3}\left(f_{a}\right) d f_{a},
$$

where

$$
\varphi_{3}=f_{a}^{3} \varphi_{0} .
$$

Finally, by integrating Eq. (30), it evolves that

$$
R_{x}=\frac{\alpha_{0}}{b} \int_{0}^{f_{a}} \varphi_{3}\left(f_{a}\right) d f_{a}=\frac{\alpha_{0}}{b} \chi\left(f_{a}\right) .
$$

Observe now that

$$
\begin{aligned}
& f_{a}=\frac{\bar{U}_{a}}{U^{*}}=\frac{\bar{U}_{a}}{\left(\tau_{p} / \rho_{p}\right)^{1 / 2}}=\left(\frac{2 \bar{\rho}_{p}}{c_{r} \bar{\rho}_{a}}\right)^{1 / 2}=\left[2 \gamma\left(1-u_{a}^{2}\right) / c_{r}\right]^{1 / 2}, \\
& \frac{\nu_{p}}{\bar{\nu}_{a}}=\left(\frac{T_{p}}{\bar{T}_{a}}\right)^{1+n}=\frac{1}{\gamma^{1+n}} \frac{1}{\left(1-u_{a}^{2}\right)^{n+1}},
\end{aligned}
$$


and let $\bar{R}_{x}=\bar{U}_{a} x / \bar{\nu}_{a}$. Then we find that

$$
\bar{R}_{x}=R_{x} \frac{\nu_{p}}{\bar{\nu}_{a}}=R_{x} \frac{1}{\gamma^{1+n}}\left(\frac{1}{1-u_{a}^{2}}\right)^{1+n}
$$

which, in conjunction with the first of Eqs. (32) and Eq. (31), establishes the relationship being sought between the Reynolds number of the undisturbed stream and the coefficient of local friction.

8. Relationship between the coefficient of total friction and the Reynolds number. The total friction drag for a length $x$ of the flat surface, is given by

$$
\begin{aligned}
F_{a} & =\int_{0}^{x} \tau_{p} d x=\int_{0}^{x} \bar{\rho}_{p} U^{*^{2}} d x=\bar{\rho}_{p} \int_{0}^{f_{a}} \frac{\bar{U}_{a}^{2}}{f_{a}^{2}} \frac{\nu_{p}}{\bar{U}_{a}} \frac{\alpha_{0}}{b} f_{a}^{3} \varphi_{0}\left(f_{a}\right) d f_{a} \\
& =\bar{\rho}_{p} \nu_{p} \bar{U}_{a} \frac{\alpha_{0}}{b} \int_{0}^{f_{a}} \varphi_{1}\left(f_{a}\right) d f_{a}=\mu_{p} \bar{U}_{a} \frac{\alpha_{0}}{b} \psi\left(f_{a}\right),
\end{aligned}
$$

where

$$
\varphi_{1}=f_{a} \varphi_{0} \quad \text { and } \quad \psi=\int_{0}^{f_{a}} \varphi_{1}\left(f_{a}\right) d f_{a} .
$$

Consequently, one deduces that

$$
C_{r}=\frac{2 F_{a}}{\bar{\rho}_{p} \bar{U}_{a}^{2} x}=\frac{2 F_{a}}{\mu_{p} \bar{U}_{a} R_{x}}=2 \frac{\psi\left(f_{a}\right)}{\chi\left(f_{a}\right)}
$$

or also that

$$
\bar{C}_{r}=\frac{2 F_{a}}{\bar{\rho}_{a} U_{a}^{2} x}=\frac{\bar{\rho}_{p}}{\bar{\rho}_{a}} C_{r}=\gamma\left(1-u_{a}^{2}\right) C_{r}=2 \gamma\left(1-u_{a}^{2}\right) \frac{\psi}{\chi} .
$$

9. Law of variation of the thickness of the boundary layer. From Eq. $\left(22^{\prime}\right)$, keeping in mind Eq. (26), one obtains for $y=\delta$,

$$
\begin{gathered}
\alpha_{0}\left[-\frac{1}{f^{*}} \frac{1}{u^{*}\left(\gamma^{1 / 2}-\beta f_{1}\right)} \log \left(1-\gamma^{1 / 2} u^{*} \frac{f_{a}}{f_{1}}\right)+\frac{1}{f^{*} u^{*}} \frac{1}{\gamma^{1 / 2}+\beta f_{2}} \log \left(1+\gamma^{1 / 2} u^{*} \frac{f_{a}}{f_{2}}\right)\right. \\
\left.+\frac{1}{u^{*}\left(\gamma^{1 / 2}+\beta f_{2}\right)\left[\left(\gamma^{1 / 2} / \beta\right)-f_{1}\right]} \log \left(1-\beta u^{*} f_{a}\right)\right] \\
=\log \left(1+b \frac{\delta U_{a}^{*}}{\nu_{p}}\right)=\log \left(1+\frac{b}{f_{a}} R_{\delta}\right),
\end{gathered}
$$

where we have set $R_{\delta}=\delta \bar{U}_{a} / \nu_{p}$. It is thus found that

$$
1+\frac{b}{f_{a}} R_{\delta}=\left(\frac{1+\gamma^{1 / 2} u_{a} / f_{2}}{1-\gamma^{1 / 2} u_{a} / f_{1}}\right)^{\alpha_{1} f_{a}}\left(\frac{1-\beta u_{a}}{1-\gamma^{1 / 2} u_{a} / f_{1}}\right)^{\alpha_{2} f_{a}},
$$

and as, in addition, one has

$$
\bar{R}_{\delta}=\frac{\delta \bar{U}_{a}}{\nu_{a}}=R_{\delta} \frac{1}{\gamma^{1+n}} \frac{1}{\left(1-u_{a}^{2}\right)^{1+n}},
$$


Eqs. (36) and $\left(36^{\prime}\right)$ give the parametric representation of the line constituting the edge of the boundary layer.

10. Explicit formulation of the drag law and of the law of variation of the thickness of the boundary layer.

10.1. Numerical results. In order to be able to give an explicit formulation of the drag law, one must, first of all, evaluate the integral defining the function $\varphi\left(f_{a}\right)$.

TABLE I.

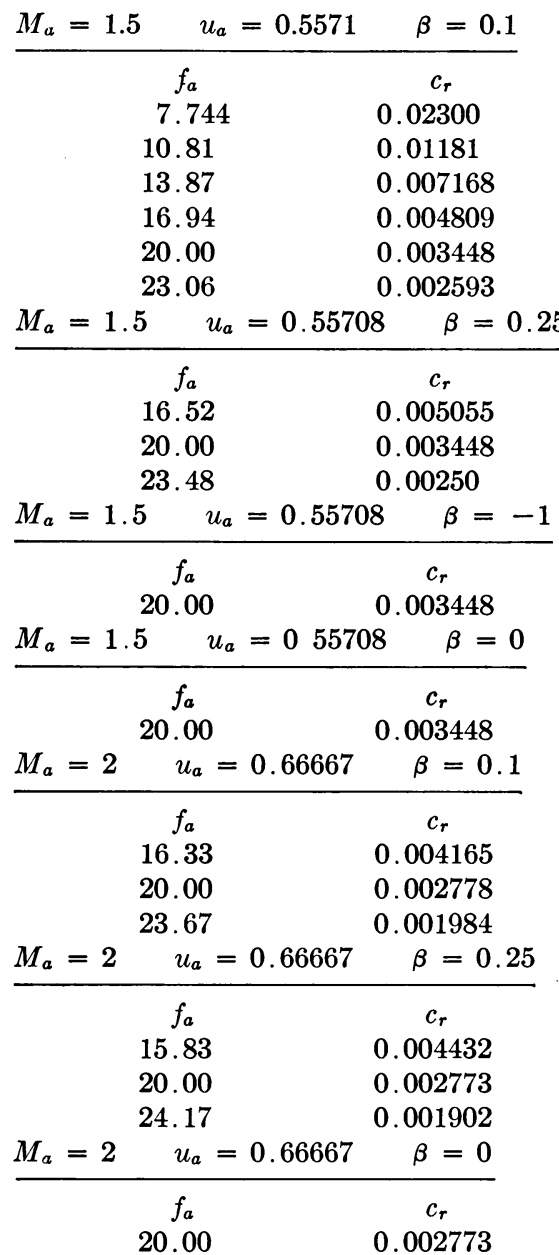

\section{$\varphi_{0}$ \\ 7.83 \\ 26.37 \\ 92.37 \\ 332.1 \\ 1216.0}

4514.0

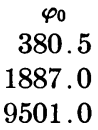

$\varphi_{0}$

140.0

$\varphi_{0}$

934.0

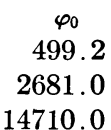

$\varphi_{0}$

844.0

6960.0

59450.0

$\varphi_{0}$
1875.0

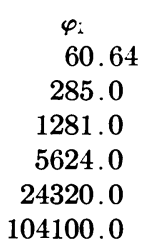

$d \bar{R}_{x} / d c_{r}$

$-5.274 \times 10^{4}$

$-13.07 \times 10^{5}$

$-2.055 \times 10^{7}$

$-2.446 \times 10^{8}$

$-2.424 \times 10^{9}$

$-2.539 \times 10^{10}$

$d \bar{R}_{x} / d c_{r}$

$-2.413 \times 10^{8}$

- $3.762 \times 10^{9}$

$-4.973 \times 10^{10}$

223110.0

$\varphi_{1}$

2801.0

$d \bar{R}_{x} / d c_{r}$

$-2.798 \times 10^{8}$

$\varphi_{1}$

18680.0

$d \bar{R}_{x} / d c_{r}$

$-18.620 \times 10^{8}$

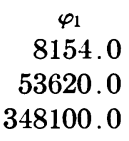

$\varphi_{1}$
13360.0
139200.0
1437000.0

$\varphi_{1}$

37500.0 $d \bar{R}_{x} / d c_{r}$

- $5.343 \times 10^{8}$

$-9.672 \times 10^{9}$

$-1.457 \times 10^{11}$

$d \bar{R}_{x} / d c_{r}$

$-7.496 \times 10^{8}$

$-25.11 \times 10^{9}$

$-6.667 \times 10^{11}$

$d \bar{R}_{x} / d c_{r}$

$-6.765 \times 10^{9}$

Since it appears impossible to work out a closed-form expression suitable for the numerical computation of this integral, the following procedure has been followed, which permits the establishment, at least qualitatively, of the drag law and of the law of variation of the thickness of the boundary layer. Their quantitative determination will be made possible only after experimentation is undertaken to arrive at the value of the parameter $\beta$ introduced above. In order to obtain a rough estimate of such a value $\beta$, reference is here made to the above-mentioned experimental results given by Frössel which deal, 
however, with friction along tubes rather than along plates. The simple case of a thermally insulated wall, $\gamma=1$, is now considered. The function $\varphi_{0}$ then takes the form

$$
\begin{aligned}
\varphi_{0}=\int_{0}^{1} & \frac{\sigma(1-\sigma)}{\left(1-u_{a}^{2} \sigma^{2}\right)\left(1-\beta u_{a} \sigma\right.}\left(\frac{1+u_{a} \sigma}{1-u_{a} \sigma}\right)^{\alpha_{1} f_{a}}\left(\frac{1-\beta u_{a} \sigma}{1-u_{a} \sigma}\right)^{\alpha_{a} a} \\
& \times\left[2+\alpha_{1} f_{a} \log \frac{1+\dot{u}_{a} \sigma}{1-u_{a} \sigma}+f_{a} \alpha_{2} \log \frac{1-\beta u_{a} \sigma}{1-u_{a} \sigma}\right] d \sigma .
\end{aligned}
$$

The values of this function, for different values of $\beta$ and of $f_{a}$ corresponding to $M_{a}=1.5$ and $M_{a}=2$, have been calculated numerically as shown in the Appendix.

\begin{tabular}{|c|c|c|c|}
\hline$M_{a}=1.5$ & & $\begin{array}{l}\text { TABLE II. } \\
\beta=0.1\end{array}$ & \\
\hline & $\left(c_{r}\right)^{-1 / 2}$ & $c_{r}^{5 / 2} d \bar{R}_{x} / d c_{r}$ & $c_{r}^{5 / 2}\left(d R_{x} / d c_{r}\right)_{i}$ \\
\hline & 6.594 & 4.231 & 3.989 \\
\hline & 9.202 & 19.81 & 19.91 \\
\hline & 11.81 & 89.39 & 94.14 \\
\hline & 14.42 & 392.30 & 397.00 \\
\hline & 17.03 & 1692.00 & 1950.00 \\
\hline & 19.64 & 7262.00 & 8694.00 \\
\hline$M_{a}=1.5$ & & $\beta=0.25$ & \\
\hline & $\left(c_{r}\right)^{-1 / 2}$ & $c_{r}^{5 / 2} d \vec{R}_{x} / d c_{r}$ & $c_{r}{ }^{5 / 2}\left(d R_{x} / d c_{r}\right)_{i}$ \\
\hline & 14.06 & 438.4 & $\begin{array}{r}352.8 \\
\end{array}$ \\
\hline & 17.03 & 2627.0 & 1950.0 \\
\hline & 19.99 & 15560.0 & 10852.0 \\
\hline$M_{a}=2$ & & $\beta=0.1$ & \\
\hline & $\left(c_{r}\right)^{-1 / 2}$ & $\overline{c_{r}{ }^{5 / 2} d \bar{R}_{x} /} d c_{r}$ & $c_{r}^{5 / 2}\left(d R_{x} / d c_{r}\right)_{i}$ \\
\hline & 15.51 & 594.8 & 804.8 \\
\hline & 18.97 & 3933.0 & 6030.0 \\
\hline & 22.45 & 25536.0 & 44337.0 \\
\hline$M_{a}=2$ & & $\beta=0.25$ & \\
\hline & $\left(c_{r}\right)^{-1 / 2}$ & $\overline{c_{r}{ }^{5 / 2} d \bar{R}_{x} / d} c_{r}$ & $c_{r}^{5 / 2}\left(d R_{x} / d c_{r}\right)_{i}$ \\
\hline & 15.02 & 980.3 & 615.4 \\
\hline & 18.97 & 10210.0 & 6030.0 \\
\hline & 22.92 & 105400.0 & 58490.0 \\
\hline$M_{a}=1.5$ & & $\beta=-1$ & \\
\hline & $\left(c_{r}\right)^{-1 / 2}$ & $\overline{c_{r}^{5 / 2} d \bar{R}_{x} / d c_{i}}$ & \\
\hline & 17.03 & 195.4 & \\
\hline & & $\beta=0$ & \\
\hline & 17.03 & 1300.0 & \\
\hline$M_{a}=2$ & & $\beta=0$ & \\
\hline & $\left(c_{r}\right)^{-1 / 2}$ & $c_{r}^{5 / 2} d \bar{R}_{x} / d c_{r}$ & \\
\hline & 18.97 & 2751.0 & \\
\hline
\end{tabular}
The results obtained are summarized in Table I, where the values of $d \bar{R}_{x} / d c_{r}$ calculated

with Eq. (30), are also given. These values have been evaluated by taking into account the second of Eqs. (32) and by assuming for $\alpha_{0}$ and for $b$ the values 0.4 and 8.93, respectively (these values are very close to those determined by Prandtl ${ }^{6}$ in his investigation). 
On the basis of the above-tabulated data, the values of $\left(c_{r}\right)^{-1 / 2}$ and of $c_{r}^{5 / 2}\left(d \bar{R}_{x} / d c_{r}\right)$ have been calculated. They are given in Table II where, in addition, the values of $c_{r}^{5 / 2}\left(d R_{x} / d c_{r}\right)$ for the same values of $c_{r}$, but for an incompressible fluid, are also given. These quantities have been calculated by means of the following equation,

$$
c_{r}^{5 / 2}\left(\frac{d R_{x}}{d c_{r}}\right)_{i}=-\frac{2^{1 / 2}}{b}\left[\exp \left\{\alpha_{0}\left(2 / c_{r}\right)^{1 / 2}\right\}\left(1-\frac{\left(2 c_{r}\right)^{1 / 2}}{\alpha_{0}}+\frac{c_{r}}{\alpha_{0}^{2}}\right)-\frac{c_{r}}{\alpha_{0}^{2}}\right],
$$

which can be obtained as a limiting case from Eq. (30). It is also possible to deduce it from the formulas given by Prandtl. ${ }^{6}$

The values of $\left(c_{r}\right)^{-1 / 2}$ and of $c_{r}^{5 / 2}\left|d \bar{R}_{x} / d c_{r}\right|$ tabulated above, are plotted on semilogarithmic scales in Fig. 1. It may be noticed at once not only that such a diagram $\left[\log \left(c_{r}^{5 / 2}\left|d \bar{R}_{x} / d c_{r}\right|\right) ; c_{r}^{-1 / 2}\right]$, referring to an incompressible fluid, approaches very closely a straight line within a very large interval, but also that the same situation holds with still higher approximation for the diagrams referring to a compressible fluid, and equally well for $M_{a}=1.5$ and $M_{a}=2$ as for $\beta=0.1$ and $\beta=0.25$.

Therefore, it seems allowable in all cases to substitute for Eq. (30) the Eq. (38) which follows. This equation holds at least for values of the Mach number not differing considerably from those given above:

$$
c_{r}^{-1 / 2}=A \log \left(c_{r}^{5 / 2}\left|\frac{d \bar{R}_{x}}{d c_{r}}\right|\right)+B .
$$

Note here that $A$ and $B$ are parameters which eventually become functions of the Mach number only. From the diagrams in Fig. 1, we infer, by setting $A^{\prime}=\left(\log _{10} e\right) A=$ $0.43429 \mathrm{~A}$, that

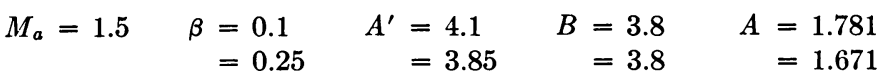

$$
\begin{aligned}
& M_{a}=2 \quad \beta=0.1 \quad A^{\prime}=4.25 \quad B=3.7 \quad A=1.846 \\
& =0.25=3.91 \quad=3.27=1.698
\end{aligned}
$$

For an incompressible fluid, in the interval corresponding to the highest values of $\left(c_{r}\right)^{-1 / 2}$, one finds $A^{\prime}=4.075 ; B=3.625 ; A=1.7697$.

10.2. Drag law. From Eq. (38) the drag law may be derived at once. In fact, let $A \log B_{0}=B$, then Eq. (38) may be written in the form

$$
c_{r}^{-1 / 2}=A \log \left(B_{0} c_{r}^{5 / 2}\left|\frac{d \bar{R}_{x}}{d c_{r}}\right|\right),
$$

whence

$$
B_{0} c_{r}^{5 / 2}\left|\frac{d \bar{R}_{x}}{d c_{r}}\right|=\exp \left(1 / A c_{r}^{1 / 2}\right)
$$

or

$$
\bar{R}_{x}=-\frac{1}{B_{0}} \int_{\infty}^{c_{r}} \exp \left(1 / A c_{r}^{1 / 2}\right) \frac{d c_{r}}{c_{r}^{5 / 2}}=\frac{2 A}{B_{0} c_{r}} \exp \left(1 / A c_{r}^{1 / 2}\right)\left(1-2 A c_{r}^{1 / 2}+2 A^{2} c_{r}\right)-\frac{4 A^{3}}{B_{0}} ;
$$

also, for $\bar{R}_{x}$ sufficiently large and hence for $c_{r}$ small enough,

$$
c_{r}^{-1 / 2}=\sim A \log \left(\bar{R}_{x} c_{r}\right)+A \log \frac{B_{0}}{2 A} .
$$


Therefore it may be stated that: for supersonic fluid flow, at least for Mach numbers not too different from those here considered, a law of resistance holds which is formally identical with that valid for incompressible fluids (logarithmic law of Kármán). It follows, consequently, that the relationship between the total and the local friction coefficients is the same as that given by Kármán for incompressible fluids, i.e.,

$$
\bar{C}_{r}=c_{r}\left(1+2 A c_{r}^{1 / 2}\right) .
$$

A quantitative determination requires the knowledge of the values of $A$ and of $B_{0}$, i.e., of $\beta$. Now the experimental results obtained by Frössel concerning fluid flow through pipes, while confirming that the logarithmic drag law holds also at supersonic speeds, give, moreover, exactly the same values of $A$ and $B_{0}$ for Mach numbers larger than unity as for the case of incompressible fluids. Assuming this result to be true also for the fluid flow past a flat plate, one may carry out the determination of the parameter $\beta$ from the data given in Fig. 1, where the results obtained for a given value of $c_{r}$ for $\beta=0$ and $\beta=-1$, are also plotted. It follows that the value of $\beta$ for any Mach number, at least for those not very different from those considered above, must lie between 0.1 and 0.25. A further rough estimate of $\beta$ will now be obtained by imposing the condition that the various curves, related to different $M_{a}$, pass through the same point of the $\left[\log \left(c_{r}^{5 / 2}\left|d \bar{R}_{x} / d c_{r}\right|\right) ; c_{r}^{-1 / 2}\right]$ plane and by supposing it to be permissible to interpolate,

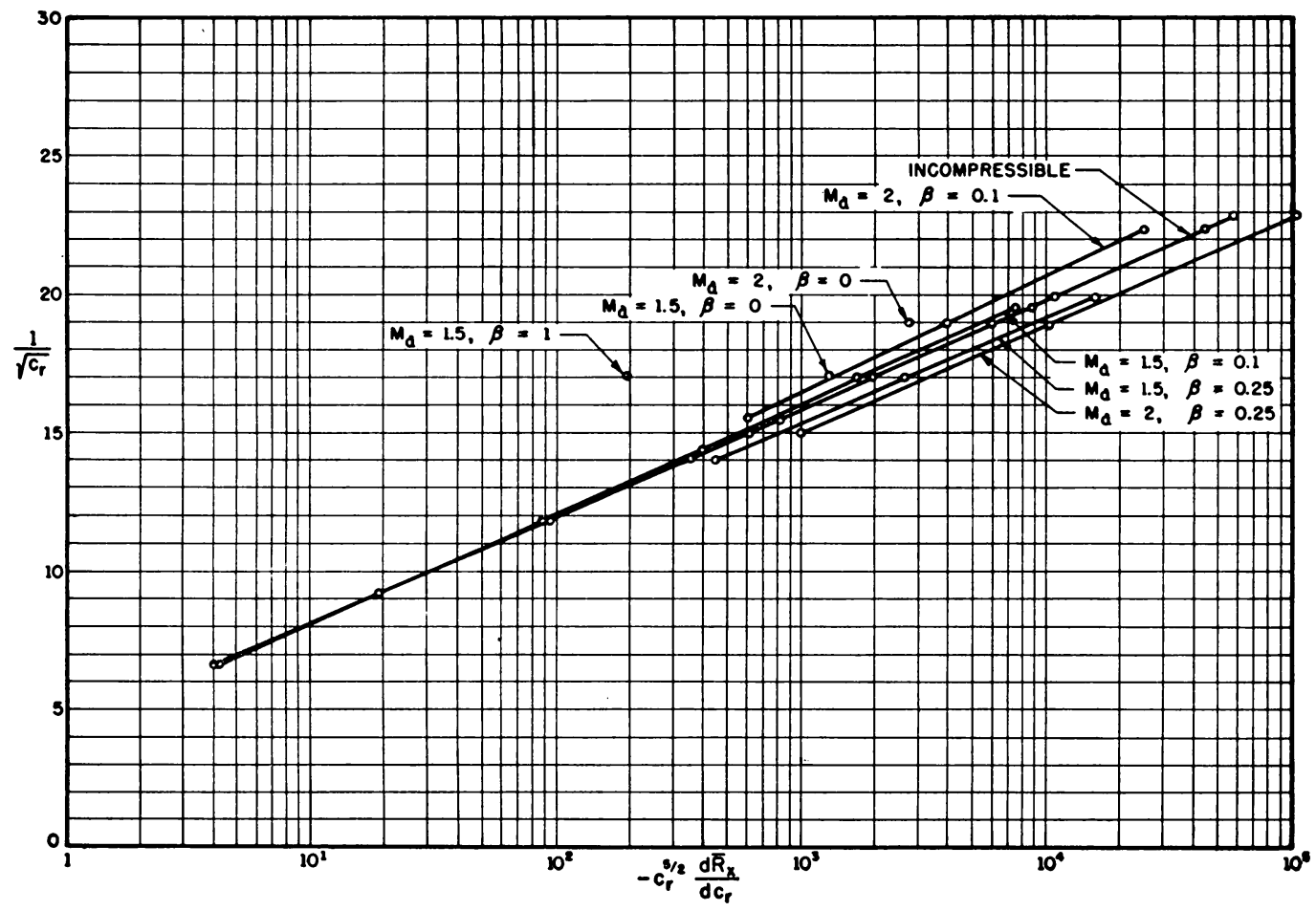

FIG. 1. Mach number influence in shifting the semi-logarithmic plots connecting the drag coefficient and Reynolds number.

for the values of $\beta$ within the given interval, between the values obtained for $\beta=0.1$ and $\beta=0.25$. 
Thus, by insisting that, for $M_{a}=1.5$ and $c_{r}^{-1 / 2}=17.0286$ (corresponding to $f_{a}=20$ ), the curve go through the point which is the ordinate of the curve representing the case of an incompressible fluid, one obtains by interpolation between the values given in Table II that $\beta=0.14$. Analogously, by imposing the condition that for $c_{r}^{-1 / 2}=18.973$ (corresponding to the same value $f_{a}=20$ ), the curve related to $M_{a}=2$ should pass through the point of the curve representing the case of $M_{a}=0$ which corresponds to the above ordinate, one obtains by interpolation between the values tabulated in Table II that $\beta=0.15$.

Figure 2 presents the graphs describing the law of variation of $c_{r}^{-1 / 2}$ in terms of the $\log \left(c_{r}^{5 / 2}\left|d \bar{R}_{x} / d c_{r}\right|\right)$ for the Mach numbers $M_{a}=1.5$ and $M_{a}=2$ and for the value of $\beta=0.145$. They have been obtained by linear interpolation of the values corresponding, for the above Mach numbers, to $\beta=0.1$ and $\beta=0.25$ respectively.

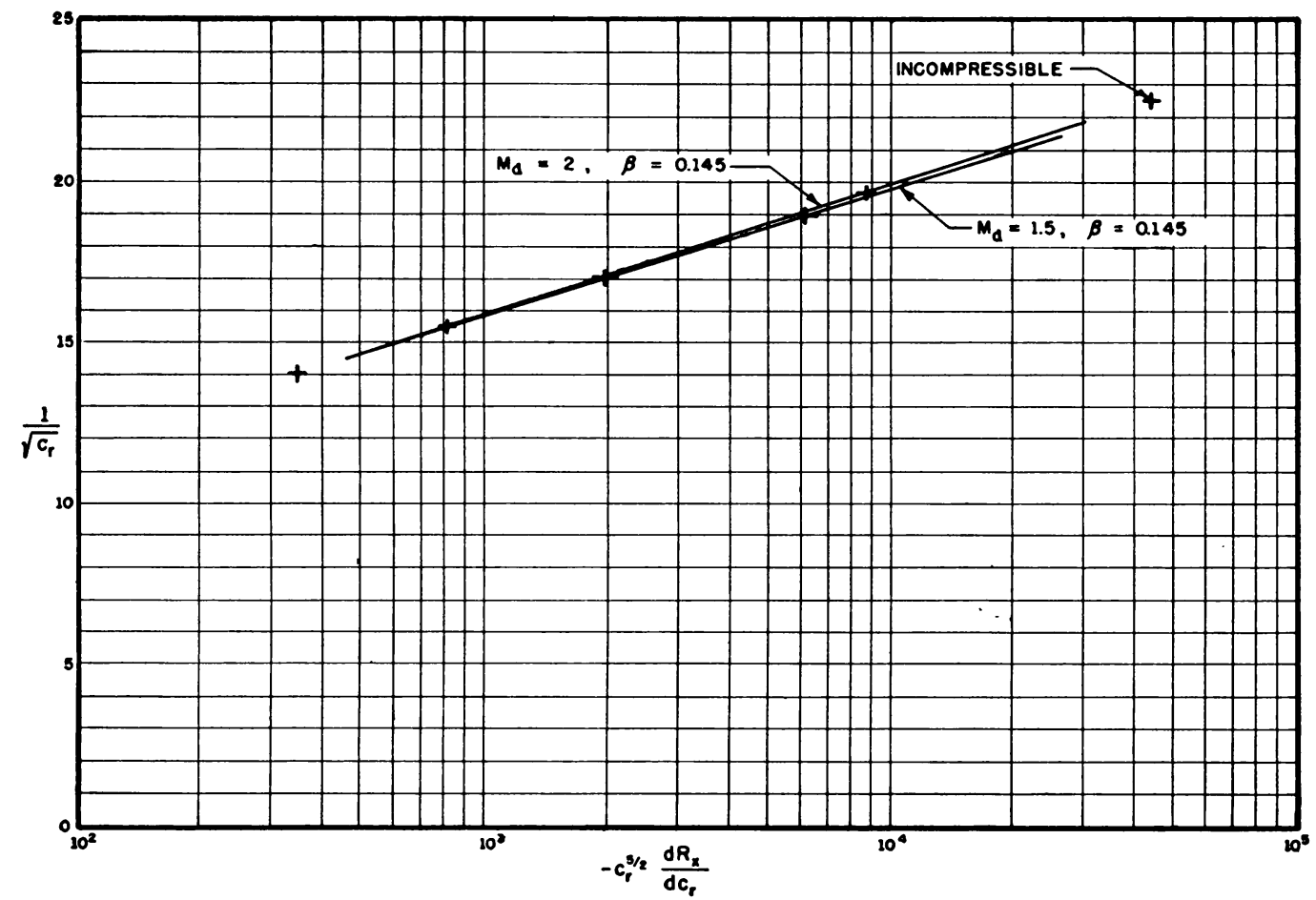

FIG. 2. Law of variation of $\left(c_{r}\right)^{-1 / 2}$ in terms of $\log \left(c_{r}^{5 / 2}\left|d \bar{R}_{x} / d c_{r}\right|\right)$ for the Mach numbers $M_{a}=1.5$ and $M_{a}=2$ and for the value of $\beta=0.145$.

It may easily be verified that:

1. The diagrams are really very close together and also close to the graph representing the case of an incompressible fluid flow (the points of which are denoted on Fig. 2 by crosses).

2. The drag coefficient decreases with increasing Mach number. This decrease may scarcely be perceived, however, on the semilogarithmic scales, for values of the Mach number varying between 1.5 and 2 .

Therefore, the assumption that the value of $\beta$ depends only in very slight measure on the Mach number seems to be plausible. This justifies the procedure followed here 
and warrants the use of expression (26) as well. It should be noted, however, that this property is a consequence of the assumption made as to the validity of the experimental data given by Frössel and their extensibility to the case of a flat plate; obviously, therefore, it deserves confirmation.*

10.3. Law of variation of the thickness of the boundary layer. For the purpose of establishing an explicit relationship interconnecting the thickness of the boundary layer, the coefficient of local resistance, and the corresponding local Reynolds number, calculations have been carried out by means of Eq. (36). For the calculations we have used $M_{a}=1.5$ and the values of $f_{a}$ and of $\beta$ already given in Sec. 10.1, which determine the values of $\bar{R}_{\delta}$ and of $\bar{R}_{\delta} c_{r}^{1 / 2}$. The results obtained are summarized in Table III. In this table, for every $c_{r}$, the corresponding value of $R_{\delta} c_{r}^{1 / 2}$ in the case of an incompressible fluid is also given; it has been calculated by means of the equation

$$
c_{r}^{1 / 2} R_{\delta}=\frac{2^{1 / 2}}{b}\left(\exp \left[\alpha_{0}\left(2 / c_{r}\right)^{1 / 2}-1\right) .\right.
$$

$M_{a}=1.5 \quad \beta=0.1$

\begin{tabular}{c}
$c c_{r}$ \\
0.02300 \\
0.01181 \\
0.007168 \\
0.004809 \\
0.003448 \\
0.002593 \\
$M_{a}=1.5 \quad \beta=0.25$ \\
\hline$c_{r}$ \\
0.05055 \\
0.003448 \\
0.002501
\end{tabular}

0.002501

TABLE III.

$\begin{array}{cc}\bar{R}_{\delta} & \bar{R}_{\delta}\left(c_{r}\right)^{1 / 2} \\ 59.52 & 9.027 \\ 352.9 & 38.35 \\ 1893.0 & 160.30 \\ 9625.0 & 667.50 \\ 47290.0 & 2777.00 \\ 226800.0 & 11550.0 \\ & \\ \bar{R}_{\delta} & \bar{R}_{\delta}\left(c_{r}\right)^{1 / 2} \\ 9983.0 & 709.8 \\ 70220.0 & 4124.0 \\ 478800.0 & 23950.0\end{array}$

The values of $\left(c_{r}\right)^{-1 / 2}$ and of $c_{r}^{1 / 2} \bar{R}_{\delta}$ are plotted on the semilogarithmic scale in Fig. 3. It may easily be seen again that not only in the case of an incompressible fluid, but also, with the same approximation and for any value of $\beta$, in the case of gaseous flow it is allowable to take

$$
c_{r}^{-1 / 2}=D \log \left(\bar{R}_{\delta} c_{r}^{1 / 2}\right)+E,
$$

where $D$ and $E$ denote parameters which are eventually functions of the Mach number only. From Fig. 3 one has

$$
\begin{aligned}
& M_{a}=1.5: \quad \beta=0.1 \\
& D^{\prime}=\log _{10} e D=4.17 \\
& =0.25 \\
& =3.805 \\
& E=2.65 \\
& D=1.81 \\
& =3.19 \\
& =1.65 \\
& M_{a}=0 \text { : } \\
& D^{\prime}= \\
& =4.04 \\
& E=3.39 \\
& D=1.75
\end{aligned}
$$

*In the light of recent experimental results for a flat plate the theoretical treatment presented here has been improved by the author. The new analysis confirms that there is very appreciable decrease in drag coefficient with increase in Mach number, and it is found necessary to select a smaller value of $\beta$, near zero, for this flow condition, in contrast to what is found for pipes. 
Following a procedure analogous to that indicated by Kármán, ${ }^{5}$ the relation being sought may be derived from Eq. (41) and Eq. (39). Subtracting Eq. (39) from Eq. (41), and observing that the values of $D$ and of $A$ are sensibly equal, therefore putting $D=$ $A$, one obtains

$$
\delta=\exp \left\{-\frac{E-B^{\prime}}{A}\right\} x c_{r}^{1 / 2},
$$

where $B^{\prime}=A \log \left(B_{0} / 2 A\right)$. Hence, the law of variation of the thickness of the boundary layer is also formally analogous to that holding for incompressible fluids. As far as the

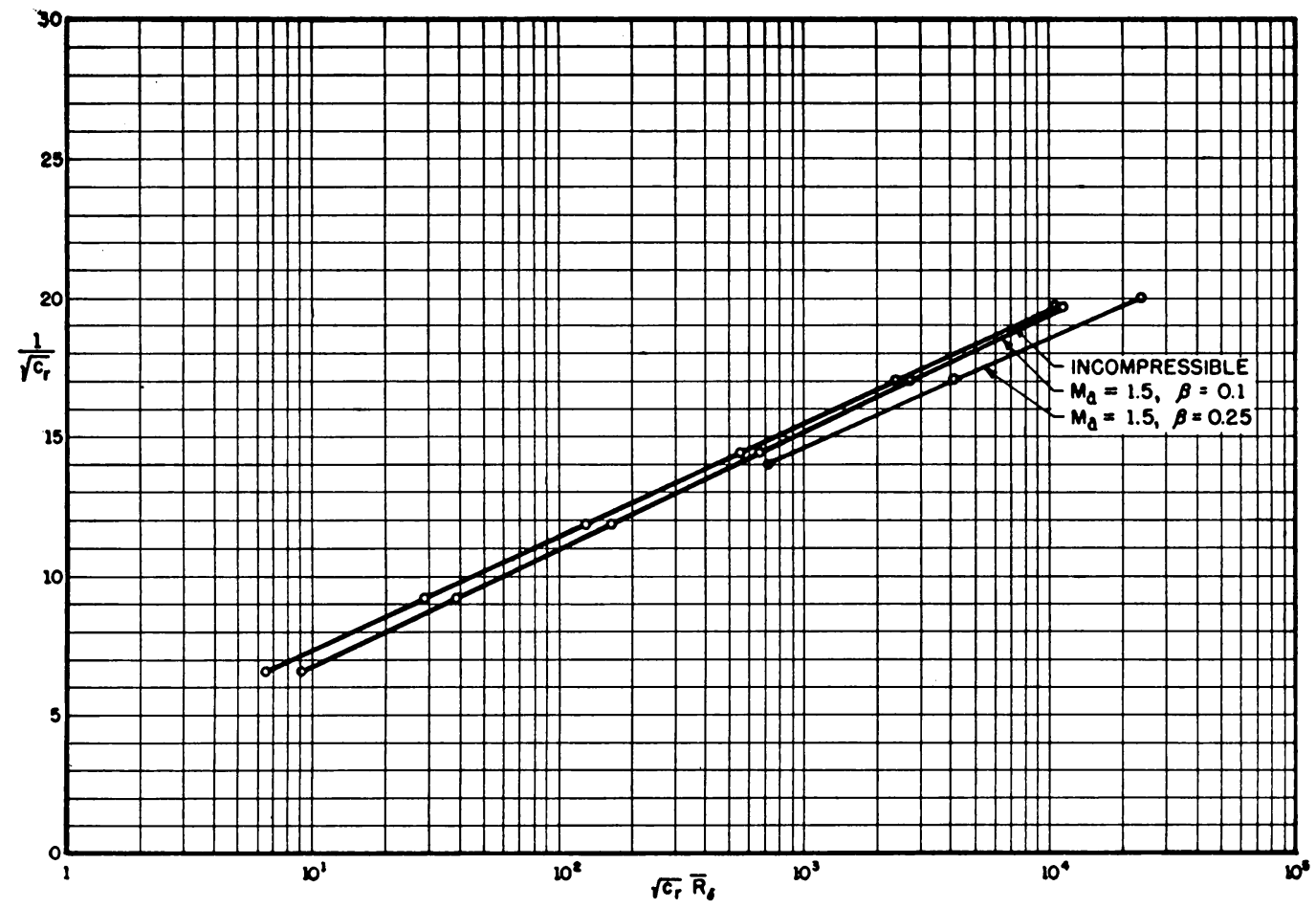

FIG. 3. Boundary layer thickness in relation to drag coefficient

quantitative determination is concerned, it is interesting to see what results correspond to the above values of $A, B, E$ in both cases, i.e., for $\beta=0.1$ and $\beta=0.25$ as well as for $M_{a}=1.5$ and $M_{a}=0$ (incompressible fluid). One has

$$
\begin{array}{ll}
M_{a}=0, \quad B^{\prime}=1.388, \quad & \exp \left\{-\frac{E-B^{\prime}}{A}\right\}=0.3226, \quad \text { whence } \delta=0.3226 x c_{r}^{1 / 2} ; \\
M_{a}=1.5, \quad \beta=0.1, \quad & B^{\prime}=1.539, \\
& \exp \left\{-\frac{E-B^{\prime}}{A}\right\}=0.5354, \quad \text { whence } \delta=0.5354 x c_{r}^{1 / 2} ;
\end{array}
$$


$M_{a}=1.5, \quad \beta=0.25, \quad B^{\prime}=1.784$,

$$
\exp \left\{-\frac{E-B^{\prime}}{A}\right\}=0.431, \quad \text { whence } \delta=0.431 x c_{r}^{1 / 2} \text {. }
$$

These results show that when $\beta$ lies between 0.1 and 0.25 , the thickness of the boundary layer grows more rapidly than in the case of incompressible fluid flow. Assuming as a probable value of $\beta$ the previously given one, $\beta=0.14$, and admitting that it is permissible to interpolate, for $\beta$ lying within the above-mentioned interval, between the values of $\delta / x c_{r}^{1 / 2}$ that we have found, one deduces the following law of variation of the thickness of the boundary layer $\left(M_{a}=1.5\right)$ :

$$
\delta=0.5 x c_{r}^{1 / 2} \text {. }
$$

Note: The results now attained have been obtained assuming $\gamma=1$, but from the qualitative point of view they still hold for $\gamma \neq 1$. A quantitative determination would require that the calculations be repeated, retaining for $\beta$ the value now deduced.

10.4. Determination of an asymptotic expression for calculating the function $\varphi_{0}\left(f_{a}\right)$. The result obtained in Sec. 10.1 suggests the determination of an asymptotic expression (holding, that is, at sufficiently large values of $f_{a}$ ), for the calculation of the function $\varphi_{0}\left(f_{a}\right)$. In this way the result is justified analytically, and we get a general expression which permits us to investigate the influence of the different parameters (Mach number, ratio of absolute temperatures, $\gamma$ ) on the drag law and on the law of variation of the thickness of the boundary layer. Such an asymptotic expression may be obtained as follows.

The integral describing $\varphi_{0}\left(f_{a}\right)$ may be written in the form

$$
\begin{aligned}
\varphi_{0}=\int_{0}^{1} & \frac{\sigma(1-\sigma)}{\left(1-\gamma^{1 / 2} \sigma u_{a} / f_{1}\right)^{2}\left(1+\gamma^{1 / 2} \sigma u_{a} / f_{2}\right)^{2}\left(1-\beta u_{a} \sigma\right)}\left[2+f_{a}\left(\alpha_{1} \log \frac{1+\gamma^{1 / 2} \sigma u_{a} / f_{2}}{1-\gamma^{1 / 2} \sigma u_{a} / f_{1}}\right.\right. \\
& \left.\left.+\alpha_{2} \log \frac{1-\beta u_{a} \sigma}{1-\gamma^{1 / 2} \sigma u_{a} / f_{1}}\right)\right] \exp \left\{f _ { a } \left[\alpha_{1} \log \frac{1+\gamma^{1 / 2} \sigma u_{a} / f_{2}}{1-\gamma^{1 / 2} \sigma u_{a} / f_{1}}\right.\right. \\
& \left.\left.+\alpha_{2} \log \frac{1-\beta u_{a} \sigma}{1-\gamma^{1 / 2} \sigma u_{a} / f_{1}}\right]\right\} d \sigma .
\end{aligned}
$$

Let

$$
\sigma=1-\epsilon
$$

one finds that

$$
\begin{aligned}
& \log \left(1+\gamma^{1 / 2} \frac{u_{a}}{f_{2}} \sigma\right)=\log \left[\left(1+\gamma^{1 / 2} \frac{u_{a}}{f_{2}}\right)\left(1-\gamma^{1 / 2} \frac{u_{a}}{f_{2}} \frac{\epsilon}{1+\gamma^{1 / 2} u_{a} / f_{2}}\right)\right] \\
& =\log \left(1+\gamma^{1 / 2} \frac{u_{a}}{f_{2}}\right)-\gamma^{1 / 2} \frac{u_{a}}{f_{2}} \frac{\epsilon}{1+\gamma^{1 / 2} u_{a} / f_{2}}-\frac{1}{2} \gamma \frac{u_{a}^{2}}{f_{2}^{2}} \frac{\epsilon^{2}}{\left(1+\gamma^{1 / 2} u_{a} / f_{2}\right)^{2}}-\cdots \\
& =\log \left(1+\gamma^{1 / 2} \frac{u_{a}}{f_{2}}\right)-\gamma^{1 / 2} \frac{u_{a}}{f_{2}} \frac{\epsilon}{1+\gamma^{1 / 2} u_{a} / f_{2}} g_{1}(\epsilon)
\end{aligned}
$$


where $g_{1}(\epsilon)$ is a function of $\epsilon$, which, as $\epsilon$ varies within the interval 0 to 1 , remains finite, continuous, and positive, and which is equal to unity for $\epsilon=0$.

Analogously, one can write

$$
\left.\begin{array}{l}
\log \left(1-\gamma^{1 / 2} \frac{u_{a}}{f_{1}} \sigma\right)=\log \left(1-\gamma^{1 / 2} \frac{u_{a}}{f_{1}}\right)+\gamma^{1 / 2} \frac{u_{a}}{f_{1}} \frac{\epsilon}{1-\gamma^{1 / 2} u_{a} / f_{1}} g_{2}(\epsilon), \\
\log \left(1-\beta u_{a} \sigma\right)=\log \left(1-\beta u_{a}\right)+\frac{\beta u_{a}}{1-\beta u_{a}} \epsilon g_{3}(\epsilon),
\end{array}\right\}
$$

where $g_{2}(\epsilon)$ and $g_{3}(\epsilon)$ are functions which satisfy the same conditions as does $g_{1}(\epsilon)$. It is then true that

$$
\begin{aligned}
& \exp \left\{f_{a}\left[\alpha_{1} \log \frac{1+\gamma^{1 / 2} \sigma u_{a} / f_{2}}{1-\gamma^{1 / 2} \sigma u_{a} / f_{1}}+\alpha_{2} \log \frac{1-\beta u_{a} \sigma}{1-\gamma^{1 / 2} \sigma u_{a} / f_{1}}\right]\right\} \\
& \quad=\left(\frac{1+\gamma^{1 / 2} u_{a} / f_{2}}{1-\gamma^{1 / 2} u_{a} / f_{1}}\right)^{\alpha_{1} f_{a}}\left(\frac{1-\beta u_{a}}{1-\gamma^{1 / 2} u_{a} / f_{1}}\right)^{\alpha_{2} f_{a}} \exp \left\{-f_{a} \phi(\epsilon) \epsilon\right\}
\end{aligned}
$$

where

$$
\phi(\epsilon)=\frac{\gamma^{1 / 2} u_{a} / f_{2}}{1+\gamma^{1 / 2} u_{a} / f_{2}} \alpha_{1} g_{1}+\frac{\gamma^{1 / 2} u_{a} / f_{1}}{1-\gamma^{1 / 2} u_{a} / f_{1}} \alpha_{3} g_{2}-\frac{\beta u_{a}}{1-\beta u_{a}} \alpha_{2} g_{3}
$$

and

$$
\alpha_{3}=\alpha_{1}+\alpha_{2} .
$$

Within the range of values of $\beta$ given in Sec. 10.2 and for $\epsilon$ varying between 0 and 1 , $\phi(\epsilon)$ is always positive.

Furthermore one has

$$
\begin{aligned}
& 2+f_{a}\left(\alpha_{1} \log \frac{1+\gamma^{1 / 2} \sigma u_{a} / f_{2}}{1-\gamma^{1 / 2} \sigma u_{a} / f_{1}}+\alpha_{2} \log \frac{1-\beta u_{a} \sigma}{1-\gamma^{1 / 2} \sigma u_{a} / f_{1}}\right) \\
& =2+f_{a}\left(\alpha_{1} \log \frac{1+\gamma^{1 / 2} u_{a} / f_{2}}{1-\gamma^{1 / 2} u_{a} / f_{1}}+\alpha_{2} \log \frac{1-\beta u_{a}}{1-\gamma^{1 / 2} u_{a} / f_{1}}\right)-f_{a} \phi(\epsilon) \epsilon ; \\
& \left(1+\gamma^{1 / 2} \frac{u_{a}}{f_{2}} \sigma\right)^{-2}=\frac{1}{\left[1+\gamma^{1 / 2} u_{a} / f_{2}\right]^{2}} \exp \left\{2 \gamma^{1 / 2} \frac{u_{a}}{f_{2}} \frac{1}{1+\gamma^{1 / 2} u_{a} / f_{2}} \epsilon g_{1}(\epsilon)\right\}, \\
& \left(1-\gamma^{1 / 2} \frac{u_{a}}{f_{1}} \sigma\right)^{-2}=\frac{1}{\left[1-\gamma^{1 / 2} u_{a} / f_{1}\right]^{2}} \exp \left\{-2 \gamma^{1 / 2} \frac{u_{a}}{f_{1}} \frac{1}{1-\gamma^{1 / 2} u_{a} / f_{1}} \epsilon g_{2}(\epsilon)\right\}, \\
& \left(1-\beta u_{a} \sigma\right)^{-1}=\frac{1}{1-\beta u_{a}} \exp \left\{-\frac{\beta u_{a}}{1-\beta u_{a}} \epsilon g_{3}(\epsilon)\right\} .
\end{aligned}
$$

Thus Eq. (44) becomes

$$
\begin{array}{r}
\varphi_{0}=\frac{1}{\gamma^{2}\left(1-u_{a}^{2}\right)^{2}\left(1-\beta u_{a}\right)}\left(\frac{1+\gamma^{1 / 2} u_{a} / f_{2}}{1-\gamma^{1 / 2} u_{a} / f_{1}}\right)^{\alpha_{1} f_{a}}\left(\frac{1-\beta u_{a}}{1-\gamma^{1 / 2} u_{a} / f_{1}}\right)^{\alpha_{2} f_{a}} \\
\times \int_{0}^{1} \epsilon(1-\epsilon) \exp \left[-\epsilon\left(f_{a} \phi+\phi_{1}\right)\right]\left[2+f_{a} F-f_{a} \epsilon \phi\right] d \epsilon,
\end{array}
$$


where one has put

$\phi_{1}=2 \gamma^{1 / 2} \frac{u_{a}}{f_{1}} \frac{1}{1-\gamma^{1 / 2} u_{a} / f_{1}} g_{2}(\epsilon)+\frac{\beta u_{a}}{1-\beta u_{a}} g_{3}(\epsilon)-2 \gamma^{1 / 2} \frac{u_{a}}{f_{2}} \frac{1}{1+\gamma^{1 / 2} u_{a} / f_{2}} g_{1}(\epsilon)$

$F=\alpha_{1} \log \frac{1+\gamma^{1 / 2} u_{a} / f_{2}}{1-\gamma^{1 / 2} u_{a} / f_{1}}+\alpha_{2} \log \frac{1-\beta u_{a}}{1-\gamma^{1 / 2} u_{a} / f_{1}}$.

In order to evaluate the integral in the right-hand member of $\left(44^{\prime}\right)$, the interval 0 to 1 may be divided into two intervals, 0 to $\eta$ and $\eta$ to 1 , where

$$
\eta=f_{a}^{-2 / 3} \text {. }
$$

Let $H$ be the maximum value attained by the expression $\epsilon(1-\epsilon)\left[2+f_{a} F-f_{a} \epsilon \phi\right]$ within the interval $\eta$ to 1 . It is found that

$$
\begin{gathered}
\int_{\eta}^{1} \epsilon(1-\epsilon)\left[2+f_{a} F-f_{a} \epsilon \phi\right] \exp \left[-\epsilon\left(f_{a} \phi+\phi_{1}\right)\right] d \epsilon<H \int_{\eta}^{1} \exp \left[-\epsilon\left(f_{a} \phi+\phi_{1}\right)\right] d \epsilon \\
=\frac{H}{f_{a} \phi+\phi_{1}}\left\{\exp \left[-\left(\phi f_{a}^{1 / 3}+\phi_{1} f_{a}^{-2 / 3}\right)\right]-\exp \left[-\left(\phi f_{a}+\phi_{1}\right)\right]\right\}=O\left(\exp \left[-\phi f_{a}^{1 / 3}\right]\right),
\end{gathered}
$$

which can be neglected for $f_{a}$ sufficiently large. In the interval $0 \leq \epsilon \leq \eta \operatorname{let} \phi=\phi_{0}+\psi_{0} \epsilon$ and $\phi_{1}=\phi_{1,0}+\psi_{1} \epsilon$. We have then that

$$
\exp \left[-\epsilon\left(f_{a} \phi+\phi_{1}\right)\right]=\exp \left[-\left(f_{a} \phi_{0}+\phi_{1,0}\right) \epsilon\right] \exp \left[-\left(f_{a} \psi_{0}+\psi_{1}\right) \epsilon^{2}\right] .
$$

Now, if we denote by $\psi_{m, 0}$ and by $\psi_{m, 1}$ the maximum absolute values that the $\psi$ may reach within the above interval, it is found that

$$
\begin{aligned}
& \exp [\left.-\left(f_{a} \phi_{0}+\phi_{1,0}\right) \epsilon\right] \exp \left[-\psi_{m, 0} f_{a}^{-1 / 3}-\psi_{m, 1} f_{a}^{-4 / 3}\right] \\
& \quad \leq \exp \left[-\epsilon\left(f_{a} \phi+\phi_{1}\right)\right] \leq \exp \left[-\left(f_{a} \phi_{0}+\phi_{1,0}\right) \epsilon\right] \exp \left[\psi_{m, 0} f_{a}^{-1 / 3}+\psi_{m, 1} f_{a}^{-4 / 3}\right]
\end{aligned}
$$

or

$$
\exp \left[-\epsilon\left(f_{a} \phi+\phi_{1}\right)\right]=\left[1+O\left(f_{a}^{-1 / 3}\right)\right] \exp \left[-\epsilon\left(f_{a} \phi_{0}+\phi_{1,0}\right)\right],
$$

and hence that

$$
\begin{aligned}
\int_{0}^{\eta} \epsilon(1-\epsilon)\left[2+f_{a} F-f_{a} \epsilon \phi\right] \exp \left[-\epsilon\left(f_{a} \phi+\phi_{1}\right)\right] d \epsilon \\
=\left[1+O\left(f_{a}^{-1 / 3}\right)\right] \int_{0}^{\eta} \epsilon(1-\epsilon)\left[2+f_{a} F-f_{a} \epsilon \phi_{0}\right] \exp \left[-\epsilon\left(f_{a} \phi_{0}+\phi_{1,0}\right)\right] d \epsilon \\
=\left[1+O\left(f_{a}^{-1 / 3}\right)\right] \frac{1}{f_{a} \phi_{0}} \frac{F}{\phi_{0}} .
\end{aligned}
$$

One thus obtains the expression being sought, namely,

$$
\left(\varphi_{0}\right)=\frac{1}{\gamma^{2}\left(1-u_{a}^{2}\right)^{2}\left(1-\beta u_{a}\right)}\left(\frac{1+\gamma^{1 / 2} u_{a} / f_{2}}{1-\gamma^{1 / 2} u_{a} / f_{1}}\right)^{\alpha_{1} f_{a}}\left(\frac{1-\beta u_{a}}{1-\gamma^{1 / 2} u_{a} / f_{1}}\right)^{\alpha_{2} f_{c}} \frac{F}{f_{a} \phi_{0}^{2}},
$$

where $\left(\varphi_{0}\right)$ denotes the asymptotic expression for $\varphi_{0}$. 
It should be noted that the following is true:

$$
\phi_{0}=\frac{\gamma^{1 / 2} u_{a} / f_{2}}{1+\gamma^{1 / 2} u_{a} / f_{2}} \alpha_{1}+\frac{\gamma^{1 / 2} u_{a} / f_{1}}{1-\gamma^{1 / 2} u_{a} / f_{1}} \alpha_{3}-\frac{\beta u_{a}}{1-\beta u_{a}} \alpha_{2}=\frac{\alpha_{0}}{\gamma\left(1-u_{a}^{2}\right)\left(1-\beta u_{a}\right)}
$$

and consequently

$$
\left(\varphi_{0}\right)=\frac{1-\beta u_{a}}{\alpha_{0}^{2}} \frac{F}{f_{a}}\left(\frac{1+\gamma^{1 / 2} u_{a} / f_{2}}{1-\gamma^{1 / 2} u_{a} / f_{1}}\right)^{\alpha_{1} f_{a}}\left(\frac{1-\beta u_{a}}{1-\gamma^{1 / 2} u_{a} / f_{1}}\right)^{\alpha_{2} f_{a}} .
$$

In order to check Eq. (52'), we compare the value of $\varphi_{0}$ given in Sec. 10.1 for $M_{a}=2$, $\beta=0.1, f_{a}=20, \gamma=1$, with that obtained from Eq. $\left(52^{\prime}\right)$.

One sees that

$$
\alpha_{1}=\frac{0.4}{2(0.66667)(1.1)}=0.273, \quad \alpha_{2}=\frac{(0.4)(0.1)}{(0.66667)(0.99)}=0.0605
$$

and hence from the second of Eqs. (49) that

$$
F=0.273 \log \frac{1.66667}{0.3333}+0.0605 \log \frac{0.9333}{0.3333}=0.501 .
$$

Remembering that

$$
\left(\frac{1+u_{a}}{1-u_{a}}\right)^{\alpha_{1} f_{a}}=\left(\frac{1.66667}{0.3333}\right)^{5.4545}=6494.3, \quad\left(\frac{1-\beta u_{a}}{1-u_{a}}\right)^{\alpha_{2} f_{a}}=\left(\frac{0.9333}{0.3333}\right)^{4.2424}=3.4834 \text {, }
$$

it follows that the value of $\left(\varphi_{0}\right)$ given by Eq. $\left(52^{\prime}\right)$ is

$$
\left(\varphi_{0}\right)=\frac{0.9333}{0.16} \cdot \frac{0.501}{20}(6494.3)(3.4834)=3290 \text { instead of } 2681 .
$$

One may improve the approximation by taking $0.99 u_{a}$ instead of $u_{a}$ in the exponentials in the right-hand side of Eq. (52'); in fact, this assumption leads to the following value for $\left(\varphi_{0}\right):\left(\varphi_{0}\right)=2820$. Analogously, for $M_{a}=2, \beta=0.1, f_{a}=23.6666$, one obtains $\left(\varphi_{0}\right)=14640$ instead of $\varphi_{0}=14707$, etc.

10.5. Justification of the logarithmic drag law. By means of Eq. (52') one can easily justify the logarithmic law for the drag, as given in Sec. 10.1. Remembering the first of Eqs. (32) in Sec. 7, we may obtain from Eq. (30) of the same section that

$$
\begin{gathered}
d R_{x}=-\frac{\alpha_{0}}{b} 2 \gamma^{2} c_{r}^{-3}\left(1-u_{a}^{2}\right)^{2}\left(\varphi_{0}\right) d c_{r}=-\frac{\alpha_{0}}{b} 2 \gamma^{2} c_{r}^{-3}\left(1-u_{a}^{2}\right)^{2} \frac{1-\beta u_{a}}{\alpha_{0}^{2}} F \\
\times \frac{c_{r}^{1 / 2} d c_{r}}{(2 \gamma)^{1 / 2}\left(1-u_{a}^{2}\right)^{1 / 2}}\left(\frac{1+\gamma^{1 / 2} u_{a} / f_{2}}{1-\gamma^{1 / 2} u_{a} / f_{1}}\right)^{\alpha_{1} f_{a}}\left(\frac{1-\beta u_{a}}{1-\gamma^{1 / 2} u_{a} / f_{1}}\right)^{\alpha_{2} f_{a}}=\gamma^{1+n}\left(1-u_{a}^{2}\right)^{1+n} d \bar{R}_{x}
\end{gathered}
$$

or

$$
\begin{aligned}
c_{r}^{5 / 2} \frac{d \bar{R}_{x}}{d c_{r}}=- & \frac{2^{1 / 2}}{\alpha_{0} b} \gamma^{0.5-n}\left(1-u_{a}^{2}\right)^{0.5-n}\left(1-\beta u_{a}\right) F \\
& \times\left(\frac{1+\gamma^{1 / 2} u_{a} / f_{2}}{1-\gamma^{1 / 2} u_{a} / f_{1}}\right)^{\alpha_{1} f_{a}}\left(\frac{1-\beta u_{a}}{1-\gamma^{1 / 2} u_{a} / f_{1}}\right)^{\alpha_{2} / a} .
\end{aligned}
$$


We find from Eq. (54) that

$$
\begin{aligned}
c_{r}^{-1 / 2}= & \frac{1}{\left[2 \gamma\left(1-u_{a}^{2}\right)\right]^{1 / 2} F} \log \left(-c_{r}^{5 / 2} \frac{d \bar{R}_{x}}{d c_{r}}\right)-\frac{1}{\left[2 \gamma\left(1-u_{a}^{2}\right)\right]^{1 / 2} F} \\
& \quad \times \log \left[\frac{2^{1 / 2}}{\alpha_{0} b} \gamma^{0.5-n}\left(1-u_{a}^{2}\right)^{0.5-n}\left(1-\beta u_{a}\right) F\right]=A \log \left(-c_{r}^{5 / 2} \frac{d \bar{R}_{x}}{d c_{r}}\right)+B,
\end{aligned}
$$

which has precisely the form of Eq. (38) with the added distinction that it now shows the influence that the Mach number and the thermic transmission through the wall have on the drag.

We shall now compare the values of $A$ and of $B$ given by Eq. (55) with those obtained in Sec. 10.1:

$$
\begin{aligned}
& M_{a}=1.5 ; \quad \beta=0.1 ; A=1.835 \quad \text { instead of } 1.781 ; \quad B=3.04 \quad \text { instead of } 3.8 \\
& =1.5 ; \quad=0.25 ; A=1.74 \quad \text { " } \quad \text { " } 1.671 ; B=2.96 \quad \text { " } \quad \text { " } 3.8 \\
& M_{a}=2 ; \beta=0.1 ; A=1.89 \quad \text { " “ } 1.846 ; B=2.92 \quad \text { " " } 3.7 \\
& =2 ; \quad=0.25 ; A=1.73 \quad \text { " } \quad \text { " } 1.698 ; B=2.64 \quad \text { " } \quad 3.27
\end{aligned}
$$

Finally, it should be noticed that from Eq. (36) in Sec. 9 the following result may be derived:

$$
\begin{gathered}
c_{r}^{-1 / 2}=\frac{1}{\left[2 \gamma\left(1-u_{a}^{2}\right)\right]^{1 / 2} F} \log \left(c_{r}^{1 / 2} \bar{R}_{\delta}\right)-\frac{1}{\left[2 \gamma\left(1-u_{a}^{2}\right]^{1 / 2} F\right.} \log \left[\frac{2^{1 / 2}}{b} \gamma^{-0.5-n}\right. \\
\left.\times\left(1-u_{a}^{2}\right)^{-0.5-n}\right]=D \log \left(\bar{R}_{\delta} c_{r}^{1 / 2}\right)+E
\end{gathered}
$$

where 1 has been neglected in comparison with $\left(b / f_{a}\right) R_{\delta}$.

On account of Eq. (42) in Sec. 10.3 and because of the expressions for $A, B, D, E$ given by Eq. (55) and Eq. (56), it is consequently found that

$$
\delta=\frac{\alpha_{0}}{2 \gamma\left(1-u_{a}^{2}\right)\left(1-\beta u_{a}\right) F} x c_{r}^{1 / 2},
$$

which clearly points out the increase in thickness of the boundary layer effected by an increase in Mach number.

\section{HEAT TRANSMISSION BETWEEN FLUID AND PLATE}

The amount of heat which is transferred from the fluid to the plate, per unit length, is given by 


$$
\begin{aligned}
q & =-\frac{\partial}{\partial x} \int_{0}^{\delta} \bar{\rho} \bar{U}\left[\bar{i}+\frac{1}{2} \bar{U}^{2}-\left(\bar{i}_{a}+\frac{1}{2} \bar{U}_{a}^{2}\right)\right] d y \\
& =-\frac{\partial}{\partial x} \int_{0}^{\delta} \bar{\rho} \bar{U} B\left(\bar{U}-\bar{U}_{a}\right) d y=B \tau,
\end{aligned}
$$

where $B$ is obtained from Eq. (10).

Therefore, what has already been stated in Sec. 5 appears to be true, i.e., that if $q=0$ then $B=0$.

On the other hand (see Kármán $\left.{ }^{5}\right) q=\lambda_{p}(d \bar{T} / d y)_{p}$, where $\lambda_{p}$ denotes the coefficient of thermal conductivity at the wall; and since from Eq. (11) one has that $(d \bar{T} / d y)_{p}=$ $\left(T_{0} / \bar{U}_{a}\right)(1-1 / \gamma)(d \bar{U} / d y)_{p}$ while $(d \bar{U} / d y)_{p}=\tau / \mu_{p}=\bar{\rho}_{a} \bar{U}_{a}^{2} c_{r} / 2 \mu_{p}$, it is found that

$$
q=\lambda_{p} \frac{T_{0}}{\bar{U}_{a}} \frac{\gamma-1}{\gamma} \frac{1}{2} \frac{\bar{\rho}_{a}}{\mu_{p}} \bar{U}_{a}^{2} c_{r}=T_{0} \bar{\rho}_{a} \bar{U}_{a} \frac{\gamma-1}{2 \gamma} c_{r} \frac{\lambda_{p}}{\mu_{p}} .
$$

The total amount of heat transferred from the fluid to the wall, for a length $x$ of the plate is then

$$
Q=\int_{0}^{x} q d x=\lambda_{p} \frac{\bar{\mu}_{a}}{\mu_{\nu}} T_{0} \frac{\bar{\rho}_{a} \bar{U}_{a} x}{\bar{\mu}_{a}} \frac{\gamma-1}{2 \gamma} \bar{C}_{r}=\lambda_{p}\left(1-u_{a}^{2}\right)^{n} \gamma^{n} T_{0} R_{x} \frac{\gamma-1}{2 \gamma} \bar{C}_{r},
$$

which is consistent with the formula given by Kármán in his paper, ${ }^{5}$ except for the different expression for $\bar{C}_{\text {r }}$.

\section{APPENDIX}

11. Sample calculation of $\varphi_{0}$ for $M_{a}=1.5, \beta=0$, and $f_{a}=20$.

$$
\varphi_{0}=\int_{0}^{1} \sigma(1-\sigma) \frac{1}{\left(1-u_{a}^{2} \sigma^{2}\right)^{2}}\left(\frac{1+u_{a} \sigma}{1-u_{a} \sigma}\right)^{\alpha_{o} f_{a} / 2 u_{a}}\left[2+\frac{f_{a} \alpha_{0}}{2 u_{a}} \log \frac{1+u_{a} \sigma}{1-u_{a} \sigma}\right] d \sigma,
$$

where

$$
\begin{aligned}
u_{a} & =\left(\frac{1}{1+2 / M_{a}^{2}(k-1)}\right)^{1 / 2} \\
& =0.5571
\end{aligned}
$$

and

$$
\frac{\alpha_{0} f_{a}}{2 u_{a}}=7.1800 .
$$

The numerical work necessary for evaluating the integral is carried out in Table IV. Column 17 gives the value of the integrand for the various values of the integration variable. Summing this column according to the trapezoidal rule gives

$$
\varphi_{0}=934.0 \text {. }
$$


TABLE IV.

\begin{tabular}{l|c|c|c|c|c|c|c|c|c}
\hline \hline & 1 & 2 & 3 & 4 & 5 & 6 & 7 & 8 & 9 \\
\hline$\sigma$ & $1-\sigma$ & $u_{a} \sigma$ & $(2)^{2}$ & $1-(3)$ & $(4)$ & 11/(5) & $1+(2)$ & $1-(2)$ & $(7 / 8)$ \\
\hline 0.0 & 1.0000 & 0 & 0 & 1.0000 & 1.0000 & 1.0000 & 1.0000 & 1.0000 & 1.0000 \\
0.1 & 0.9000 & 0.0557 & 0.0031 & 0.9969 & 0.9938 & 0.9056 & 1.0557 & 0.9443 & 1.1180 \\
0.2 & 0.8000 & 0.1114 & 0.0124 & 0.9876 & 0.9754 & 0.8202 & 1.1114 & 0.8886 & 1.2507 \\
0.3 & 0.7000 & 0.1671 & 0.0279 & 0.9721 & 0.9450 & 0.7407 & 1.1671 & 0.8329 & 1.4012 \\
0.4 & 0.6000 & 0.2228 & 0.0497 & 0.9503 & 0.9031 & 0.6644 & 1.2228 & 0.7772 & 1.5733 \\
0.5 & 0.5000 & 0.2786 & 0.0776 & 0.9224 & 0.8508 & 0.5877 & 1.2786 & 0.7214 & 1.7724 \\
0.6 & 0.4000 & 0.3343 & 0.1117 & 0.8883 & 0.7891 & 0.5069 & 1.3343 & 0.6657 & 2.0055 \\
0.65 & 0.3500 & 0.3621 & 0.1311 & 0.8689 & 0.7550 & 0.4636 & 1.3621 & 0.6379 & 2.1353 \\
0.70 & 0.3000 & 0.3900 & 0.1521 & 0.8479 & 0.7189 & 0.4173 & 1.3900 & 0.6100 & 2.2787 \\
0.75 & 0.2500 & 0.4178 & 0.1746 & 0.8254 & 0.6813 & 0.3669 & 1.4178 & 0.5822 & 2.4352 \\
0.80 & 0.2000 & 0.4457 & 0.1987 & 0.8013 & 0.6421 & 0.3115 & 1.4457 & 0.5543 & 2.6082 \\
0.85 & 0.1500 & 0.4735 & 0.2243 & 0.7757 & 0.6017 & 0.2493 & 1.4735 & 0.5265 & 2.7987 \\
0.90 & 0.1000 & 0.9014 & 0.2514 & 0.7486 & 0.5604 & 0.1784 & 1.5014 & 0.4986 & 3.0112 \\
0.95 & 0.0500 & 0.5292 & 0.2801 & 0.7199 & 0.5183 & 0.0965 & 1.5292 & 0.4708 & 3.2481 \\
1.00 & 0 & 0.5571 & 0.3104 & 0.6896 & 0.4755 & 0 & 1.5571 & 0.4429 & 3.5157 \\
& & & & & & & & & \\
\hline
\end{tabular}

\begin{tabular}{|c|c|c|c|c|c|c|c|c|}
\hline & 10 & 11 & 12 & 13 & 14 & 15 & 16 & 17 \\
\hline$\sigma$ & $\log _{10}$ (9) & 7.18 (10) & $\log _{10}-1$ & (6) $\times$ (12) & $\sigma$ (13) & 2.3026 (11) & $2+(15)$ & (16) $\times$ (14) \\
\hline 0.0 & 0 & 0 & 1.0000 & 1.0000 & 0 & 0 & 2.0000 & 0 \\
\hline 0.1 & 0.04844 & 0.3478 & 2.2274 & 2.0171 & 0.2017 & 0.8008 & 2.8008 & 0.5649 \\
\hline 0.2 & 0.09716 & 0.6976 & 4.9842 & 4.0880 & 0.8176 & 1.6063 & 3.6063 & 2.948 \\
\hline 0.3 & 0.14650 & 1.0519 & 11.2695 & 8.3473 & 2.5042 & 2.4221 & 4.4221 & 11.07 \\
\hline 0.4 & 0.19681 & 1.4131 & 25.8882 & 17.2001 & 6.8800 & 3.2538 & 5.2538 & 36.15 \\
\hline 0.5 & 0.24856 & 1.7847 & 60.9114 & 35.7976 & 17.8988 & 4.1095 & 6.1095 & 111.80 \\
\hline 0.6 & 0.30198 & 2.1682 & 147.3000 & 74.6664 & 44.7998 & 4.9925 & 6.9925 & 313.00 \\
\hline 0.65 & 0.32946 & 2.3655 & 232.0053 & 107.5577 & 69.9125 & 5.4468 & 7.4468 & 520.00 \\
\hline 0.70 & 0.35768 & 2.5681 & 369.9167 & 154.3662 & 108.0563 & 5.9133 & 7.9133 & 855.00 \\
\hline 0.75 & 0.38654 & 2.7754 & 596.2143 & 218.7510 & 164.0632 & 6.3906 & 8.3906 & 1376.00 \\
\hline 0.80 & 0.41634 & 2.9893 & 975.6600 & 303.9181 & 243.1345 & 6.8832 & 8.8832 & 2159.00 \\
\hline 0.85 & 0.44696 & 3.2092 & 1618.8148 & 403.5705 & 343.0349 & 7.3895 & 9.3895 & 2534.00 \\
\hline 0.90 & 0.47874 & 3.4374 & 2737.8125 & 488.4258 & 439.5832 & 7.9150 & 9.9150 & 4358.00 \\
\hline 0.95 & 0.51163 & 3.6735 & 4715.2222 & 455.0189 & 432.2680 & 8.4586 & 10.4586 & 4520.0 \\
\hline 1.00 & 0.54601 & 3.9204 & 8325.3333 & 0 & 0 & 9.0271 & 11.0271 & 0 \\
\hline
\end{tabular}

